\title{
BUTLER GROUPS OF INFINITE RANK II
}

\author{
MANFRED DUGAS, PAUL HILL, AND K. M. RANGASWAMY
}

\begin{abstract}
A torsion-free abelian group $G$ is called a Butler group if $\operatorname{Bext}(G, T)$ $=0$ for any torsion group $T$. We show that every Butler group $G$ of cardinality $\aleph_{1}$ is a $B_{2}$-group; i.e., $G$ is a union of a smooth ascending chain of pure subgroups $G_{\alpha}$ where $G_{\alpha+1}=G_{\alpha}+B_{\alpha}, B_{\alpha}$ a Butler group of finite rank. Assuming the validity of the continuum hypothesis $(\mathrm{CH})$, we show that every Butler group of cardinality not exceeding $\aleph_{\omega}$ is a $B_{2}$-group. Moreover, we are able to prove that the derived functor $\operatorname{Bext}^{2}(A, T)=0$ for any torsion group $T$ and any torsion-free $A$ with $|A| \leq \aleph_{\omega}$. This implies that under $\mathrm{CH}$ all balanced subgroups of completely decomposable groups of cardinality $\leq \aleph_{\omega}$ are $B_{2}$-groups.
\end{abstract}

\section{INTRODUCTION}

All groups in this paper are (torsion-free) abelian groups. Undefined notations are standard as in [Fu]. A torsion-free abelian group is called a Butler group if $\operatorname{Bext}(B, T)=0$ for all torsion groups $T$. Here Bext is the subfunctor of Ext of all balanced-exact extensions. It is known [BS] that this definition coincides with the familiar one if $B$ has finite rank, i.e., a pure subgroup of a completely decomposable group. The main result in this paper is that under the continuum hypothesis $\left(\mathrm{CH}\right.$ ) each Butler group $B$ (of rank $\leq \aleph_{\omega}$ ) is a $B_{2}$-group [A]; i.e., $B$ is the union of a smooth ascending chain of pure subgroups $B_{\alpha}$, $\alpha<\lambda$ an ordinal and $B_{\alpha+1}=B_{\alpha}+L_{\alpha}$ for all $\alpha<\lambda$ where $L_{\alpha}$ is a Butler group of finite rank. This result partially answers questions raised in [BSS], [A], and elsewhere. We will, in fact, prove the following:

Theorem $[\mathrm{CH}]$. Let $G$ be a torsion-free group of cardinality $\leq \aleph_{\omega}$.

$(\alpha)$ The following are equivalent:

(1) $\operatorname{Bext}(G, T)=0$ for all torsion groups $T$.

(2) $\operatorname{Bext}(G, T)=0$ for all $\Sigma$-cyclic torsion groups $T$.

(3) $G$ is a $B_{2}$-group.

( $\beta$ ) The derived function $\operatorname{Bext}^{2}(G, T)=0$ for any torsion group $T$. (We have to use $\mathrm{CH}$ only if $|G| \geq \aleph_{2}$.)

Received by the editors October 13,1988.

1980 Mathematics Subject Classification (1985 Revision). Primary 20K20.

The research of the first author was partially supported by NSF Grant DMS 8701074 .

The research of the second author was partially supported by NSF Grant DMS 8521770 . 
Part $(\alpha)$ of the preceding theorem extends results [A], [AH], [BS], [BSS], [D], and [DR 1]. Part $(\beta)$ was shown for $|G| \leq \aleph_{1}$ in [AH]. The statement that (3) implies (1) may be found in [BS]. Under $V=L$ it was shown in [DR2], with some help from [D], that (2) implies (3) in case $|G| \leq \aleph_{1}$.

As an immediate consequence of the theorem we obtain the following:

Corollary [CH]. If $G$ is a balanced subgroup of a completely decomposable group and $|G|<\aleph_{\omega}$, then $G$ is a $B_{2}$-group.

The proof of the theorem will show that if the corollary holds in general, we may drop the cardinality restriction in the theorem.

Albrecht and Hill $[\mathrm{AH}]$ showed (without $\mathrm{CH}$ ) that the preceding corollary is true if $|G| \leq \aleph_{1}$.

In order to prove the above theorem we have to prove numerous auxiliary results, some of independent interest. These auxiliary results include the following: we show that any balanced subgroup of a completely decomposable group admits a $G\left(2^{\aleph_{0}}\right)$-family (cf. [FH]) of balanced subgroups. The proof is a modification of one in $[\mathrm{AH}]$. Using methods different from the proof of this result, we show that for any torsion-free group $G$ of cardinality $\aleph_{n}, n \leq \omega$, there is a filtration $G=\bigcup_{\alpha<\aleph_{n}} G_{\alpha}$ into pure subgroups $G_{\alpha}$ such that $G_{\alpha+1} / G_{\alpha}$ has rank 1 and each $G_{\alpha}$ is separable in $G$ in the sense of Hill; i.e., for all $g \in G$ there is a countable subset $S$ of $G_{\alpha}$ such that for any $h \in G_{\alpha}$ there is some $s=s(h)$ in $S$ for which $|g+h| \leq|g+s|$.

The main obstacle we have to overcome is the fact the pure subgroups of Butler groups (of infinite rank) are in general not again Butler groups. In order to show that balanced subgroups of Butler groups are Butler again, one needs to know that $\operatorname{Bext}^{2}(G, T)=0$ for torsion-free groups $G$. We were able to show this for all torsion-free groups $G$ of cardinality not exceeding $\aleph_{\omega}$.

If $T$ is a torsion group and $G$ a torsion-free group, any pure subgroup $A \subseteq G$ gives rise to a natural map $\operatorname{Bext}(G, T) \stackrel{i^{*}}{\rightarrow} \operatorname{Bext}(A, T)$. Under the hypothesis that $A$ is separable in $G,|G / A| \leq \aleph_{1}$, and each pure subgroup of finite rank of $G$ is Butler, we are able to show that $i^{*}$ is onto. This enables us to show that some balanced subgroups of completely decomposable groups are again Butler groups.

We will prove our theorem by cardinal induction. We slightly modify Hodges' version $[\mathrm{H}]$ of S. Shelah's singular-compactness theorem to fit our situation. The case of regular cardinals is then treated similarly to [EF, Lemma 9].

\section{Preliminaries}

Unless otherwise specified, all the groups that we consider here are additively written torsion-free abelian groups. We generally follow the notation and terminology of [Fu]. A height is a sequence $h=\left(h_{p}\right)$, indexed by the set $P$ of primes $p$ such that $h_{p}$ is an ordinal or the symbol $\infty$ for each $p$. If $G$ is an abelian group and $x \in G$, then $|x|=\left(h_{p}^{G}(x)\right)$ is a height, where $h_{p}^{G}(x)$ is the 
$p$-height of $x$ in $G$. If $S$ is a subset of a torsion-free group $G$, then $\langle S\rangle,\langle S\rangle_{*}$ denote, respectively, the subgroup and the pure subgroup generated by $S$. If $f: A \rightarrow B$ is a map and $S \subseteq A$, then $f \mid S$ denotes the restriction of $f$ to $S$. If $T$ is a torsion group, $p$ is a prime, and $x \in T$, then $T_{p}$ is the $p$-component of $T$ and $x_{p}$ the $p$-component of $x$ in $T$.

A subgroup $H$ of a torsion-free group $G$ is said to be separable [HM] if to each $g \in G$ is a countable subset $C$ of $H$ such that to each $h \in H$, there is $s \in C$ such that $|g+h| \leq|g+s|$.

A torsion-free abelian group $G$ is called a Butler group if $\operatorname{Bext}(G, T)=0$ for all torsion groups $T$. A torsion-free group $G$ is said to be a $B_{2}$-group if $G$ is the union of a smooth chain $0=G_{0} \subseteq G_{1} \subseteq \cdots \subseteq G_{\alpha} \subseteq \cdots$ of pure subgroups $G_{\alpha}$ such that, for each $\alpha, G_{\alpha+1}=G_{\alpha}+B_{\alpha}$ where $B_{\alpha}$ is a finite-rank Butler group. In this case we call $G=\bigcup G_{\alpha}$ a $B$-filtration. Let $H$ be a pure subgroup of a torsion-free group $G$. We say that $H$ satisfies the torsion extension property (TEP, for short) in $G$ or $G$ has the TEP over $H$ if, for any $\Sigma$-cyclic torsion group $T$, every homomorphism $f: H \rightarrow T$ extends to a homomorphism $g: G \rightarrow T$. In [DR2] it was shown that every pure subgroup of a finite-rank Butler group $G$ has the TEP in $G$. A subgroup $S$ of a torsion-free group $G$ is said to be decent [AH] if to each finite subset $X$ of $G$ there is a finite-rank Butler group $B$ such that $S+B$ is pure and contains $X$. An important result of [DR2] is that in a countable Butler group $G$, a pure subgroup $H$ is decent if and only if $H$ has the TEP in $G$. It is known that a $B_{2}$-group $G$ is always a Butler group [BS] and every finite-rank pure subgroup of $G$ is Butler. It is easy to see that if $H$ is a decent subgroup of $G$ and both $H$ and $G / H$ are $B_{2}$-groups, then $G$ is also a $B_{2}$-group. In the following $\mathbf{Q}$ denotes the additive group of rational numbers and $\mathbf{Z}$ the additive groups of integers. As usual, $\mathrm{CH}$ denotes the continuum hypothesis, i.e., $2^{\aleph_{0}}=\aleph_{1}$.

\section{Pure subgroups of Infinite-Rank Butler groups}

The backbone of the investigation of Butler groups in [DR2] is the theorem that in countable Butler groups the TEP over a pure subgroup implies the decency of the subgroup. In this section we generalize this important result to uncountable Butler groups. Using this, we answer in more than one way a question raised by Arnold [A] and Bican and Salce [BS] for Butler groups of rank $\aleph_{1}$, namely, whether countable pure subgroups of such groups are again Butler. Using a different approach, this was shown in [D].

We begin our investigation with the following useful lemma.

Lemma 3.1. (i) Let $G=\bigcup G_{\alpha}$ be a B-filtration of pure subgroups $G_{\alpha}$. Then $G$ has the TEP over each $G_{\alpha}$.

(ii) Suppose $G$ is a $B_{2}$-group. Then every countable subgroup $H$ is contained in a countable pure subgroup $A$ which as the TEP in $G$.

Proof. (i) Let $f: G_{\alpha} \rightarrow T$ be any homomorphism where $T$ is an arbitrary torsion group. By hypothesis, $G_{\alpha+1}=G_{\alpha}+B_{\alpha} ; B_{\alpha}$ is pure in $G$ and is a 
finite-rank Butler group. By Theorem 2 of [DR2], $f \mid\left(B_{\alpha} \cap G_{\alpha}\right): B_{\alpha} \cap G_{\alpha} \rightarrow T$ extends to a homomorphism $g: B_{\alpha} \rightarrow T$. Then $f_{\alpha+1}: G_{\alpha+1} \rightarrow T$ given by $f_{\alpha+1}(x+y)=f(x)+g(y)$ for all $x \in G_{\alpha}, y \in B_{\alpha}$ is a homomorphism extending $f$. By transfinite induction, on $\gamma \geq \alpha$, we get an extension $f^{\prime}: G \rightarrow T$.

(ii) By $[\mathrm{AH}], G$ has an axiom-3 family $\mathscr{C}$ of decent and pure subgroups. Now $H \subset A \in \mathscr{C}$, where $A$ is countable. The axiom-3 nature of $\mathscr{C}$ and the decency of $A$ enable us to build a $B$-filtration of $G$ beginning with $A$. By (i) above, $G$ has the TEP over $A$.

Lemma 3.1 is useful in proving the following generalization of Theorem 7 of [DR2].

Proposition 3.2. Let $A$ be a pure subgroup of the torsion-free group $G$ such that

(i) $A$ has TEP in $G$.

(ii) $A$ is a $B_{2}$-group.

(iii) Each finite-rank pure subgroup of $G$ is Butler.

Then $A$ is decent in $G$.

Proof. We may assume without loss of generality that $G / A$ has finite rank. Then $G=H+A$, for some countable pure subgroup $H$. Since $H \cap A$ is countable and $A$ is a $B_{2}$-group, Lemma 3.1 (ii) implies that $H \cap A \subseteq B \subseteq A$, $B$ a countable pure subgroup which has the TEP in $A$ and hence in $G$. Now $B$ has the TEP in the countable Butler group $\langle H+B\rangle_{*}$ and $\langle H+B\rangle_{*} / B$ has finite rank. Thus Theorem 7 of [DR2] applies and $\langle H+B\rangle_{*}=B_{1}+B$, where $B_{1}$ is a finite-rank Butler group. Then $G=H+A=\langle H+B\rangle_{*}+A=B_{1}+A$ and $A$ is decent in $G$.

Arnold [A] and Bican, Salce, and Stepan [BS], [BSS] raised the question whether countable pure subgroups of Butler groups are always Butler. Proposition 3.2 enables us to answer this in the affirmative for Butler groups of rank $\aleph_{1}$. Dugas [D] also answers this for groups of rank $\aleph_{1}$ using another approach.

Theorem 3.3. (i) Suppose $G=C / K$ is a Butler group where $C$ is completely decomposable and $K$ is a balanced subgroup of $C$. If $K$ is a $B_{2}$-group, then every countable pure subgroup of $G$ is Butler.

(ii) If $G$ is a Butler group of rank $\aleph_{1}$, then every countable pure subgroup of $G$ is also Butler.

Proof. (i) Let $H / K$ be a countable pure subgroup of $C / K$. Since $G$ is Butler, $K$ has the TEP in $C$ and hence in $H$. Also, any finite-rank pure subgroup of $H$ is Butler. Thus Proposition 3.2 applies and $K$ is decent in $H$. Since $K$ is balanced in $H$, we conclude that $H / K$ is the union of an increasing sequence of finite-rank Butler groups and hence is itself Butler; cf. [A].

(ii) Albrecht and Hill [AH] have shown that a balanced subgroup of a completely decomposable group of rank $\aleph_{1}$ is a $B_{2}$-group. Hence (ii) follows from (i).

As another application of Proposition 3.2, we obtain the following (not very satisfactory) characterization of $B_{2}$-groups. 
Theorem 3.4. An infinite-rank torsion-free group $G$ is a $B_{2}$-group if and only if every finite-rank pure subgroup of $G$ is Butler and $G=\bigcup G_{\alpha}$ is a smooth filtration of pure subgroups $G_{\alpha}$ where, for each $\alpha, G_{\alpha}$ has the TEP in $G$ and $G_{\alpha+1} / G_{\alpha}$ is countable.

Proof. We need only prove the sufficiency. We first claim that each $G_{\alpha}$ is a $B_{2}$-group. Suppose, by way of contradiction, there is a $\beta$ such that $G_{\beta}$ is not a $B_{2}$-group. We may assume $\beta$ is the least such ordinal. Then for each $\alpha<\beta$, $G_{\alpha}$ is a $B_{2}$-group and has the TEP in $G_{\alpha+1}$, Then, by Proposition 3.2, $G_{\alpha}$ is decent in $G_{\alpha+1}$. Since, further, $G_{\alpha+1} / G_{\alpha}^{\alpha+1}$ is countable, the filtration $\bigcup_{\alpha<\beta} G_{\alpha}$ can be refined to a $B$-filtration. This would imply that $G_{\beta}$ is a $B_{2}$-group, a contradiction. Thus each $G_{\alpha}$ be a $B_{2}$-group and, by Proposition 3.2, must be decent in $G_{\alpha+1}$, Thus the smooth filtration $G=\bigcup G_{\alpha}$ refines into a $B$-filtration of $G$, showing that $G$ is a $B_{2}$-group.

Since, for groups of cardinality $\aleph_{1}$, a smooth filtration of countable groups is an axiom-3 family, we get an axiom-3 characterization of $B_{2}$-groups in terms of pure subgroups with the TEP.

Corollary 3.5. A torsion-free group $G$ is a $B_{2}$-group if and only if $G$ satisfies the TEP over an axiom-3 family of pure subgroups and every finite-rank pure subgroup of $G$ is Butler.

Before we proceed further we review the following proposition, which is a slight recast of Lemma 10 of [DR1] and which is also implicit in the works of Hill and Megibben [HM].

Proposition 3.6. Suppose $C$ is a completely decomposable group of regular cardinal $\kappa, H$ is a balanced subgroup $C$, and $G=C / H$. Then there are $a$ closed and unbounded subset $E$ of $\kappa$ and smooth filtrations $H=\bigcup_{\alpha \in E} H_{\alpha}$, $C=\bigcup_{\alpha \in E} C_{\alpha}$, and $G=\bigcup_{\alpha \in E} G_{\alpha}$ such that, for each $\alpha \in E, H_{\alpha}$ is balanced in $H, C_{\alpha}$ is a direct summand of $C, G_{\alpha}$ is pure in $G, G_{\alpha}=\left(C_{\alpha}+H\right) / H$, $H_{\alpha}=H \cap C_{\alpha}$, and $\left|H_{\alpha}\right|=\left|C_{\alpha}\right|=\left|G_{\alpha}\right|<\kappa$.

We obtain from Proposition 3.6 an alternative proof of a stronger form of Theorem 3.3. In $\S 7$ we will prove a stronger version of 3.7.

Theorem 3.7. Let $G=C / H$ be a Butler group of regular cardinality $\kappa$, where $C$ is completely decomposable and $H$ is balanced.

(i) If $\mathrm{H}$ is a $\mathrm{B}_{2}$-group, then every countable pure subgroup of $G$ is Butler and $G$ has a smooth filtration of pure subgroups $G=\bigcup G_{\alpha}$ where each $G_{\alpha}$ is a Butler group of cardinality $<\kappa$.

(ii) If $G$ is a Butler group of rank $\aleph_{1}$, then every countable pure subgroup of $G$ is Butler.

Proof. By Proposition 3.6, there are a closed and unbounded subset $E$ of $\kappa$ and smooth filtrations $H=\bigcup_{\alpha \in E} H_{\alpha}, C=\bigcup_{\alpha \in E} C_{\alpha}$, and $G=\bigcup_{\alpha \in E} G_{\alpha}$ with the stated properties. Let $H=\bigcup_{\beta<k} L_{\beta}$ be a $B$-filtration of the $B_{2}$-group $H$, so that for all $\beta<k, L_{\beta+1}=L_{\beta}+B_{\beta}$, where $B_{\beta}$ is a Butler group of finite 
rank. By Lemma 3.1, $H$ has the TEP over each $L_{\beta}$. Now, by Eklof [E], the two filtrations $\cup L_{\beta}$ and $\cup H_{\alpha}$ agree on a closed and unbounded subset $E^{\prime}$ of $E$. Hence we may assume that the $H=\bigcup_{\alpha \in E} H_{\alpha}, C=\bigcup_{\alpha \in E} C_{\alpha}$, and $G=\bigcup_{\alpha \in E} G_{\alpha}$ satisfy the conclusion of Proposition 3.6 with the additional property that $H$ has the TEP over each $H_{\alpha}$. Since $G$ is Butler, $C$ has the TEP over $H$. Then $H$ and hence $C_{\alpha}$ will have the TEP over $H_{\alpha}$. Since $H_{\alpha}$ is a $B_{2}$-group, Proposition 3.2 implies that $H_{\alpha}$ is decent in $C_{\alpha}$. Since $H_{\alpha}$ is balanced in $C_{\alpha}$, we conclude that $G_{\alpha}$ is a Butler group in which every finite-rank pure subgroup is Butler.

A natural question is whether the converse of Theorem 3.7 (i) holds. Observe that if $G=\bigcup_{\alpha \in E} G_{\alpha}$ is a smooth filtration of pure Butler subgroup $G_{\alpha}$ and each countable pure subgroup of $G$ is Butler, then Proposition 3.6 provides a closed and unbounded subset $E^{\prime}$ of $E$ and a filtration $H=\bigcup_{\alpha \in E^{\prime}} H_{\alpha}$ where each $H_{\alpha}$ is balanced in $H, H_{\alpha}=H \cap C_{\alpha}$, and $C_{\alpha} / H_{\alpha} \cong G_{\alpha}$, so that $C_{\alpha}$ has the TEP over $H_{\alpha}$. By Proposition 3.2, $H_{\alpha}$ is decent in $C_{\alpha}$ and hence in $H$. Thus $H=\bigcup_{\alpha \in E^{\prime}} H_{\alpha}$ is a smooth filtration where, for each $\alpha, H_{\alpha}$ is balanced, decent, and has the TEP in $H$ and every countable subgroup of $H$ is Butler. Moreover, $H$ itself is decent in $C$. Is $H$ a $B_{2}$-group? When does $H$ fit into a $B$-filtration of $C$ ? These are the questions that we wish to explore next.

Lemma 3.8. Suppose $A$ and $B$ are pure subgroups of $G$ with $A \subseteq B$. If $A$ and $B / A$ have the TEP respectively in $G$ and $G / A$, then $B$ has the TEP in $G$.

Proof. Let $T$ be any torsion group and $f \in \operatorname{Hom}(B, T)$. Now $f \mid A: A \rightarrow T$ extends to a homomorphism $h: G \rightarrow T$. Since $h-f$ vanishes on $A, h-f \mid B$ induces $h_{1}: B / A \rightarrow T$, which, by hypothesis, extends to a $g_{1} \in \operatorname{Hom}(G / A, T)$. Let $g_{2}$ be the natural map $G \rightarrow G / A$ followed by $g_{1}$. Then $g=h-g_{2}$ satisfies, for all $b \in B, g(b)=h(b)-g_{2}(b)=f(b)$.

Proposition 3.9. Let $G$ be a torsion-free group such that any finite-rank pure subgroup is Butler. Suppose $A$ is a balanced subgroup or $A$ is a $B_{2}$-group. Then $A$ fits into a B-filtration of $G$; i.e., $G=\bigcup_{\alpha<\lambda} G_{\alpha}$ with $G_{0}=A, G_{\alpha+1}=G_{\alpha}+B_{\alpha}$, $B_{\alpha}$ finite-rank Butler if and only if $A$ has the TEP in $G$ and $G / A$ is a $B_{2}$-group.

Proof. Clearly, if $A$ fits into a $B$-filtration with $A=G_{0}, G_{\alpha+1}=G_{\alpha}+B_{\alpha}$, $B_{\alpha}$ finite-rank Butler, and $G=\bigcup_{\alpha<\lambda} G_{\alpha}$, then $\bigcup_{\alpha<\lambda}\left(G_{\alpha} / A\right)$ is a $B$-filtration of $G / A$ which is therefore a $B_{2}$-group. By Lemma 3.1, $A$ will have the TEP in $G$.

Conversely, Let $G / A=\bigcup_{\alpha<\lambda}\left(G_{\alpha} / A\right)$ be a $B_{2}$-filtration so that, for each $\alpha$, $G_{\alpha+1} / A=G_{\alpha} / A+B_{\alpha} / A$ where $B_{\alpha} / A$ is finite-rank Butler. If $A$ is balanced then $B_{\alpha}=A+B_{\alpha}^{\prime}$, where $B_{\alpha}^{\prime}$ is finite-rank Butler, and so $G_{\alpha+1}=G_{\alpha}+B_{\alpha}^{\prime}$, proving that $\cup G_{\alpha}$ with $G_{0}=A$ is a $B_{2}$-filtration of $G$ from $G_{0}$ onwards. If, on the other hand, $A$ is a $B_{2}$-group, then Proposition 3.2 applies so that $A$ is decent in $G$. Thus, as before, $B_{\alpha}=A+B_{\alpha}^{\prime}, B_{\alpha}^{\prime}$ finite-rank Butler. Then $\cup G_{\alpha}$ with $G_{0}=A$ is a $B_{2}$-filtration of $G$ and this second-case $G$ itself is a $B_{2}$-group. 
Corollary 3.10. Let $B=\bigcup_{\alpha<\lambda} B_{\alpha}$ be a smooth filtration with $B_{0}=0$ and, for each $\alpha, B_{\alpha}$ is pure and has the TEP in $B$. If $B_{\alpha+1} / B_{\alpha}$ is a $B_{2}$-group for all $\alpha$, then $B$ itself is $a B_{2}$-group.

The following proposition provides a partial answer to the question raised earlier.

Proposition 3.11. Suppose $0 \rightarrow H \rightarrow C \rightarrow G \rightarrow 0$ is a balanced exact sequence of torsion-free groups of cardinality $\aleph_{n}, n<\omega$. If $C$ and $G$ are $B_{2}$-groups, then so is $H$.

Proof. Identify $H$ with a subgroup of $C$ and take $G=C / H$. We will do an induction on $n$, the result being true for $n=0$. Suppose $G=\bigcup_{\alpha<\omega_{n}} G_{\alpha}$, $C=\bigcup_{\alpha<\omega_{n}} C_{\alpha}$ are $B$-filtrations of $G$ and $C$, respectively, so that for each $\alpha$, $G_{\alpha}$ and $C_{\alpha}$ are decent and have the TEP respectively in $G$ and $C$; for $\alpha<\beta$, $G_{\beta} / G_{\alpha}$ and $C_{\beta} / C_{\alpha}$ are $B_{2}$-groups, $G_{\alpha}$ and $C_{\alpha}$ are $B_{2}$-groups themselves, and $\left|C_{\alpha}\right|=\left|G_{\alpha}\right|<\aleph_{n}$. The proof of Proposition 3.6 shows that there are closed and unbounded subset $E$ of $\omega_{n}$ and smooth filtrations $H=\bigcup_{\alpha \in E} H_{\alpha}$, $C=\bigcup_{\alpha \in E} C_{\alpha} G=\bigcup_{\alpha \in E} G_{\alpha}$ such that $H_{\alpha}=H \cap C_{\alpha}, H_{\alpha}$ balanced in $C_{\alpha}$, $G_{\alpha}=\left(H+C_{\alpha}\right) / H \cong C_{\alpha} / H_{\alpha}$. Since $G_{\alpha}$ is a $B_{2}$-group (and hence Butler) and $H_{\alpha}$ is balanced in $C_{\alpha}, H_{\alpha}$ has the TEP in $C_{\alpha}$ and hence in $C$. By induction, $H_{\alpha}$ is a $B_{2}$-group for each $\alpha$. Now $H_{\alpha+1} / H_{\alpha}$ is a balanced subgroup of $C_{\alpha+1} / H_{\alpha}$. Moreover, $C_{\alpha+1} / H_{\alpha}$ is a decent extension of the $B_{2}$-group $C_{\alpha} / H_{\alpha}$ by the $B_{2}$ group $C_{\alpha+1} / C_{\alpha}$ and hence is itself a $B_{2}$-group. Since $\left(C_{\alpha+1} / H_{\alpha}\right) /\left(H_{\alpha+1} / H_{\alpha}\right) \cong$ $C_{\alpha+1} / H_{\alpha+1} \cong G_{\alpha+1}$ is a $B_{2}$-group, cardinal induction implies that $H_{\alpha+1} / H_{\alpha}$ is a $B_{2}$-group.

\section{Properties of $\operatorname{Bext}(-, T)$ and $h$-Maps}

It is well known [Hu] that if $0 \rightarrow A \stackrel{\alpha}{\rightarrow} B \stackrel{\beta}{\rightarrow} C \rightarrow 0$ is balanced exact, then for any torsion $T, 0 \rightarrow \operatorname{Hom}(C, T) \rightarrow \operatorname{Hom}(B, T) \rightarrow \operatorname{Hom}(A, T) \stackrel{\delta}{\rightarrow}$ $\operatorname{Bext}(C, T) \stackrel{\beta^{*}}{\rightarrow} \operatorname{Bext}(B, T) \stackrel{\alpha^{*}}{\rightarrow} \operatorname{Bext}(A, T)$ is exact. We want to investigate what can be said without the balancedness of $0 \rightarrow A \rightarrow B \rightarrow C \rightarrow 0$.

The concept of an $h$-map is introduced and its relationship to separable subgroups and, in particular, balanced subgroups is investigated. The notion of an $h$-map appears to have the potential to play a dominant role in the future investigations of infinite-rank Butler groups.

Lemma 4.1. Consider the following row exact commutative diagram:

$$
\begin{aligned}
& 0 \rightarrow T \rightarrow M^{\prime} \stackrel{\eta}{\longrightarrow} G \rightarrow 0 \\
& \downarrow \delta \quad \downarrow \beta \\
& 0 \rightarrow T \rightarrow M \stackrel{\alpha}{\longrightarrow} G / A \rightarrow 0
\end{aligned}
$$


where the right square is a pullback, $T$ any group and $G / A$ torsion-free. If the bottom row is balanced exact, then the top row is balanced exact.

Proof. Let $R$ be a torsion-free group of rank 1 and $\varphi: R \rightarrow G$ a homomorphism. Since the bottom row is balanced, there is a map $\psi: \beta(\varphi(R)) \rightarrow M$ with $\beta \varphi=\alpha \psi$. Thus there exists a map $\gamma: R \rightarrow M^{\prime}$ with $\eta \gamma=\varphi$ since $M^{\prime}$ is a pullback. This shows that the top row is balanced.

The following is an immediate consequence:

Corollary 4.2. The map $\beta: G \rightarrow G / A, G / A$ torsion-free, induces a map

$$
\beta^{*}: \operatorname{Bext}(G / A, T) \rightarrow \operatorname{Bext}(G, T)
$$

for any torsion group $T$.

We will need the following later:

Corollary 4.3. If $G$ is a Butler group and has the TEP over a pure subgroup $A$, then $G / A$ is a Butler group.

Proof. Since $A$ has the TEP over $G$, the following is exact:

$$
0 \rightarrow \operatorname{Ext}(G / A, T) \stackrel{\beta^{*}}{\rightarrow} \operatorname{Ext}(G, T) .
$$

Since $G / A$ is torsion-free we may apply Corollary 4.2 and obtain

$$
0 \rightarrow \operatorname{Bext}(G / A, T) \stackrel{\beta^{*}}{\rightarrow} \operatorname{Bext}(G, T)
$$

is exact. Thus $\operatorname{Bext}(G / A, T)=0$ and $G / A$ is a Butler group.

If $A \subseteq G$ is as above, we want to investigate when

$$
\operatorname{Bext}(G, T) \stackrel{\alpha^{*}}{\rightarrow} \operatorname{Bext}(A, T) \rightarrow 0
$$

is exact for some $0 \rightarrow A \stackrel{\alpha}{\rightarrow} G$. This turns out to be quite involved.

First we need some notation. Let $T$ be a reduced torsion group and $A$ reduced torsion-free. We will fix a mixed group $M$ that fits into $0 \rightarrow T \rightarrow M \stackrel{\pi}{\rightarrow}$ $A \rightarrow 0$. For any group $G$, let $\widehat{G}$ be the cotorsion hull of $G$; see [F1]. Then $G$ is a pure subgroup of $\widehat{G}$ and $\widehat{G} / G$ is divisible. We obtain a commutative diagram with exact rows:

$$
\begin{gathered}
0 \rightarrow \widehat{T} \rightarrow \widehat{M} \underset{\rho}{\stackrel{\hat{\pi}}{\rightleftarrows}} \hat{A} \rightarrow 0, \\
\cup \quad \cup \quad \cup \\
0 \rightarrow T \rightarrow M \stackrel{\pi}{\rightarrow} A \rightarrow 0 .
\end{gathered}
$$

$\hat{\pi}$ is the map induced by $\pi$ on the cotorsion hull and $\rho$ is a splitting map, i.e., $\hat{\pi} \rho=\mathrm{id}_{\widehat{A}}$. We will use this to obtain a description of $M$ as a subgroup of $\widehat{M}$ : There is a map $\varphi: A \rightarrow \widehat{T}$ such that $M=T+\langle\rho(b)+\varphi(b) \mid b \in A\rangle$. Here $\varphi: A \rightarrow \widehat{T}$ is a map such that $\varphi^{*}: A \rightarrow \widehat{T} / T$ defined by $\varphi^{*}(x)=\varphi(x)+T$ is a homomorphism. If we pick another splitting map $\rho^{\prime}: \widehat{A} \rightarrow \widehat{M}$, then again $M=$ 
$T+\left\langle\rho^{\prime}(b)+\varphi^{\prime}(b) \mid b \in A\right\rangle$ and an easy computation shows $\varphi(b) \equiv \varphi^{\prime}(b) \bmod T$ for all $b \in A$. We will call such maps $\varphi$ and $\varphi^{\prime}$ equivalent since they yield the same mixed group $M$ in the way described above. We find the maps $\varphi: A \rightarrow \widehat{T}$ to be of some help since they allow us to "code" most of the structure of $M$ into a single map. In what follows we will omit the splitting map $\rho$; i.e., we will set $\rho=\mathrm{id}_{\widehat{A}}$. Since we are interested in the elements of $\operatorname{Bext}(A, T)$ we will investigate what maps $\rho: A \rightarrow \widehat{T}$ belong to mixed groups $M$ with $T$ balanced in $M$.

Definition. Let $A$ be a torsion-free group. A map $\varphi: A \rightarrow \widehat{T}$ is called an $h$-map if

(j) The composite $a \mapsto \varphi(a)+T$ is a homomorphism from $A$ into $\widehat{T} / T$.

(ij) If $|a|_{p}=\infty$, then $\varphi(a)_{p} \in T_{p}+p^{\infty} \widehat{T}_{p}$.

(iij) For almost all primes $p,|a|_{p} \leq|\varphi(a)|_{p}$ for any $a \in A$.

We call two $h$-maps $\varphi, \psi: A \rightarrow \widehat{T}$ equivalent if for all $a \in A, \varphi(a) \equiv$ $\psi(a) \bmod T$. Note that each $h$-map is equivalent to an $h$-map $\psi$ and $|\psi(a)|_{p} \geq$ $|a|_{p}$ for all $a \in A$ and all primes $p$.

The next proposition relates $h$-maps from $A$ into $\widehat{T}$ to elements in $\operatorname{Bext}(A, T)$. There we will use the following observation: If $X, Y$ are pure subgroups of some group $G$ containing its torsion part $T$ with $X \cap Y=T$ and $Y / T$ divisible, then $X+Y$ is pure in $G$. We omit the straightforward proof.

Proposition 4.4. Let $T$ be a reduced torsion group and $B$ reduced torsion-free.

(j) If $E: 0 \rightarrow T \rightarrow M \rightarrow B \rightarrow 0$ is exact, then $E$ is balanced exact if and only if there is an h-map $\varphi: B \rightarrow \widehat{T}$ such that as a subgroup of $\widehat{M}=$ $\operatorname{Ext}(\mathbf{Q} / \mathbf{Z}, M) \cong \widehat{T} \oplus \widehat{B}, M=T+\langle\{b+\varphi(b) \mid b \in B\}\rangle$.

(jj) If the short exact sequence $E$ is balanced exact, then $E$ splits iff the $h$ map $\varphi$ is equivalent to a homomorphism $\varphi: B \rightarrow \widehat{T}$, i.e., $\varphi(b)+T=\varphi(b)+T$ for all $b \in B$.

Proof. (j) Let $E$ be balanced exact. The mixed group $M$ is a pure subgroup of its cotorsion hull $\widehat{M}=\widehat{T} \oplus \widehat{B}$. Since $\widehat{T} / T$ is divisible and $M / T$ reduced, $M \cap \widehat{T}=T$. If $\pi: \widehat{M} \rightarrow \widehat{B}$ is the natural projection along $\widehat{T}$, then $\pi(M)=B$. The remark above gives that $M+\widehat{T}$ is pure in $\widehat{M}$ and hence $B=(M+\widehat{T}) / \widehat{T}$ is pure in $\widehat{M} / \widehat{T}=\widehat{B}$. Since $T$ is balanced in $M$, to each $b \in B$ there exists $m_{b} \in M$ such that $\left|m_{b}\right|=|b|$ and $m_{b}+T=b$. We may write $m_{b}=\pi^{\prime}\left(m_{b}\right)+b$, where $\pi^{\prime}: \widehat{M} \rightarrow \widehat{T}$ is the projection. Clearly, $\left|\pi^{\prime}\left(m_{b}\right)\right| \geq\left|m_{b}\right|=|b|$. Then the map $\varphi: B \rightarrow \widehat{T}$ defined by $\varphi(b)=\pi^{\prime}\left(m_{b}\right)$ satisfies $|\varphi(b)| \geq|b|$ and the induced map $\bar{\varphi}: b \mapsto \varphi(b)+T$ is a homomorphism. Thus $\varphi$ is an $h$-map and $M=T+\left\langle m_{b}: b \in B\right\rangle=T+\langle\{\varphi(b)+b: b \in B\}\rangle$.

Conversely, suppose $0 \rightarrow T \rightarrow M \rightarrow B \rightarrow 0$ is exact and there is an $h$ map $\varphi: B \rightarrow \widehat{T}$ with $M=T+\langle\varphi(b)+b \mid b \in B\rangle \subseteq \widehat{M}$. Then $M$ is a mixed group with $M / T \cong B$. If $0 \neq b \in B$, then $|\varphi(b)|_{p} \geq|b|_{p}$ for almost all $p$. If $|\varphi(b)|_{p}<|b|_{p}$, then $|b|_{p}<\infty$ because $\varphi$ is an $h$-map. Since 
$\varphi(b)_{p} \in \widehat{T}_{p}$ is divisible $\bmod T_{p}$ and there are only finitely many such primes with $|\varphi(b)|_{p}<|b|_{p}$, we find $t \in T$ with $|t+\varphi(b)|_{p} \geq|b|_{p}$ for all primes $p$. Thus $\varphi(b)+t$ is proper in $\varphi(b)+T$ and $T$ is balanced in $M$. The proof of (jj) is straightforward.

The following theorem is crucial for this paper. It will enable us to discover many subgroups of Butler groups that are again Butler groups.

Theorem 4.5. Let $A$ be a pure and separable subgroup of the torsion-free group $B$ with $|B / A| \leq \aleph_{1}$. Let $\varphi: A \rightarrow \widehat{T}$ be an h-map and assume that each pure subgroup of finite rank of $B$ is Butler. Then there exists an h-map $\psi: B \rightarrow \widehat{T}$ with $\psi \mid A=\varphi$.

Proof. We may assume that $B / A$ is countable. For each coset $b+A \in B / A$ there exists a countable subset $C=C(b) \subseteq A$ such that for each $a \in A$ there exists an element $a_{0} \in C$ with $|b+a| \leq\left|b+a_{0}\right|$. Let $B_{0}$ be the pure and countable (!) subgroup generated by a representing system of the cosets in $B / A$ together with the countably many elements in $C=C(b), b$ running over the representing system of $B / A$. Let $A_{0}=A \cap B_{0}$. Then $A_{0}$ is pure in $A$ and our hypotheses imply that $A_{0}$ and $B_{0}$ are (countable) Butler groups. Moreover, $B=A+B_{0}$. Since $A_{0}$ is a Butler group we may apply $4.4(\mathrm{jj})$ and assume that $\varphi \mid A_{0} \in \operatorname{Hom}\left(A_{0}, \widehat{T}\right)$. Since $\widehat{T}$ is pure-injective there is a $\psi \in \operatorname{Hom}\left(B_{0}, \widehat{T}\right)$ with $\psi\left|A_{0}=\varphi\right| A_{0}$. For each pair $\left(b_{0}, a\right) \in B_{0} \times A$ we fix $a_{0}=a_{0}\left(b_{0}, a\right) \in A_{0}$ with $\left|b_{0}+a\right| \leq\left|b_{0}+a_{0}\right|$. Note that such an $a_{0} \in A_{0}$ exists by the choice of $A_{0}$.

Now define $\rho\left(b_{0}+a\right)=\psi\left(b_{0}+a_{0}\right)+\varphi\left(a-a_{0}\right)$. Then $\rho: B \rightarrow \widehat{T}$ is a map. Suppose $b=b_{0}+a \in B_{0}$. Then $a \in A_{0}$ and we have $\rho\left(b_{0}+a\right)=\psi\left(b_{0}+a_{0}\right)+$ $\varphi\left(a-a_{0}\right)=\psi\left(b_{0}+a\right)$ since $\psi$ is a homomorphism with $\psi\left|A_{0}=\varphi\right| A_{0}$. Thus $\rho \mid B_{0}=\psi$. If $b_{0}+a \in A$, then $b_{0} \in B_{0} \cap A=A_{0}$ and

$$
\begin{aligned}
\rho\left(b_{0}+a\right) & =\psi\left(b_{0}+a_{0}\right)+\varphi\left(a-a_{0}\right) \\
& =\varphi\left(b_{0}+a_{0}\right)+\varphi\left(a-a_{0}\right) \equiv \varphi\left(b_{0}+a\right) \bmod T .
\end{aligned}
$$

Thus $\rho \mid A$ is equivalent to $\varphi$ and for $M=T+\{(\varphi(a), a) \mid a \in A\}, M^{\prime}=$ $T+\{(\rho(b), b) \mid b \in B\}$ we have that the diagram

$$
\begin{gathered}
0 \rightarrow T \rightarrow M \rightarrow A \rightarrow 0 \\
\| \cap \cap \\
0 \rightarrow T \rightarrow M^{\prime} \rightarrow B \rightarrow 0
\end{gathered}
$$

is commutative. In order to finish the proof we have only to show that $\rho$ is an $h$-map. (After that we may change $\rho$ into an equivalent $h$-map $\rho^{\prime}$ with $\rho^{\prime} \mid A=\varphi$.) Let $b \in B, b=b_{0}+a, b_{0} \in B_{0}, a \in A$. Then $|b|=\left|b_{0}+a\right| \leq$ $\left|b_{0}+a_{0}\right|$ where $a_{0} \in A_{0}$. We obtain

$$
\left|\rho\left(b_{0}+a\right)\right|_{p} \geq \min \left\{\left|\psi\left(b_{0}+a_{0}\right)\right|_{p},\left|\varphi\left(a-a_{0}\right)\right|_{p}\right\} \geq\left.\min \left\{\left|b_{0}+a_{0}\right|_{p}, \mid a-a_{0}\right)\right|_{p}
$$

for almost all primes $p$. If $q$ is a prime with $\left|b_{0}+a\right|_{q}=\infty$, then $\left|b_{0}+a_{0}\right|_{q}=$ $\infty=\left|a-a_{0}\right|_{q}$ and $\varphi\left(a-a_{0}\right)_{q}, \psi\left(b_{0}+a_{0}\right)_{q} \in T_{q}+q^{\infty} \widehat{T}_{q}$. Thus $\rho\left(b_{0}+a\right)_{q} \in$ $T_{q}+q^{\infty} \widehat{T}_{q}$. This completes the proof. 
Corollary 4.6. Let $B$ be a Butler group such that for any countable subset $X$ of $B$ there is a filtration $B=\bigcup_{\alpha<\lambda} B_{\alpha}$ such that $\left|B_{\alpha+1} / B_{\alpha}\right| \leq \aleph_{1}, X \subseteq B_{0}$, $\left|B_{0}\right| \leq \aleph_{1}$, and each $B_{\alpha}$ is a separable subgroup of $B_{\alpha+1}$. Then each $B_{\alpha}$ is a Butler group.

Proof. Let $A$ be a pure subgroup of finite rank of $B$. Then we have a filtration $B=\bigcup_{\alpha<\lambda} B_{\alpha}$ such that each $B_{\alpha}$ is separable in $B_{\alpha+1}$ and $B_{0}=A$. By [D, Theorem 2] and [B] (see also [A]) we conclude that $A$ is a Butler group. Thus any pure countable subgroup of $B$ is Butler. Let $T$ be torsion and let $0 \rightarrow$ $T \rightarrow M_{\alpha} \rightarrow B_{\alpha} \rightarrow 0$ be balanced exact. By Theorem 4.5 , there is a commutative diagram

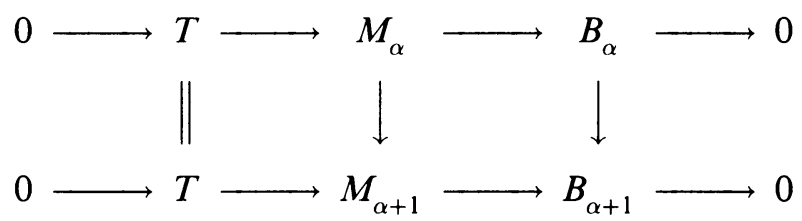

where $B_{\alpha} \rightarrow B_{\alpha+1}$ is the embedding and the bottom row is balanced exact. A transfinite induction provides a commutative diagram

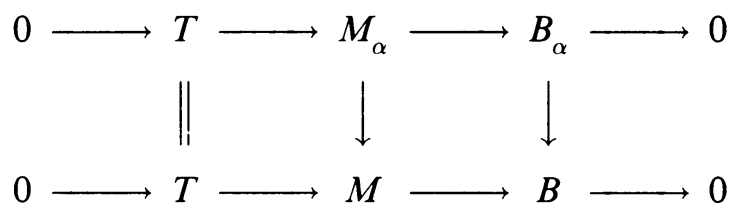

where the bottom row is balanced exact. Since $B$ is Butler, $M=T \oplus B^{\prime}$ for some subgroup $B^{\prime}$ isomorphic to $B$. Since $T \subseteq M_{\alpha}$ we obtain

$$
M_{\alpha}=M \cap M_{\alpha}=\left(T+B^{\prime}\right) \cap M_{\alpha}=T \oplus\left(B^{\prime} \cap M_{\alpha}\right) .
$$

Thus $M_{\alpha}$ splits and $B_{\alpha}$ is a Butler group.

Example. A group $B$ with pure subgroup $A, \operatorname{rk}(B / A)=1$ and there is an $h$-map $\varphi: A \rightarrow T=\prod_{p} \widehat{T}_{p}$ which does not lift to $B$.

Construction. Let $\mathscr{F}$ be a maximal family of almost disjoint maps $f: \omega \rightarrow$ $\omega$, i.e., $f \neq g \in \mathscr{F}=\{i \mid f(i)=g(i)\}$ finite.

Generators. $x, x_{n}(n<\omega), x_{f}(f \in \mathscr{F})$.

Define $B=\left\langle x, x_{n}, x_{f} \mid n<\omega, f \in \mathscr{F}\right\rangle$.

Let $\pi$ be an infinite set of primes,

$$
\pi=\left\{p_{i j} \mid i, j<\omega\right\} .
$$

Relations. $\left(x+x_{n}\right) / p_{n, i}(i<\omega)$ and $\left(x+x_{f}\right) / p_{n, f(n)}(n<\omega)$, i.e.,

$$
B=\left\langle x, x_{n}, x_{f}, \frac{x+x_{n}}{p_{n, i}}, \frac{x+x_{f}}{p_{n, f(n)}} \mid n<\omega, i<\omega, f \in \mathscr{F}\right\rangle .
$$

Let $A=\left\langle x_{n}, x_{f} \mid n<\omega, f \in \mathscr{F}\right\rangle_{*}$. Then

$$
A=\left\langle x_{n}, x_{f}, \frac{x_{n}-x_{f}}{p_{n, f(n)}} \frac{x_{f}-x_{g}}{p_{n, f(n)}} \mid n<\omega, g, f \in \mathscr{F}, f(n)=g(n)\right\rangle .
$$


Pick elements $s_{i j}, t_{i j} \in T_{p_{i j}}$ with $\left|s_{i j}-t_{i j}\right|_{p_{i j}}=0$.

Define

$$
\varphi\left(x_{n}\right)_{p_{i j}}= \begin{cases}s_{i j} & \text { if } i=n \\ 0 & \text { if } i \neq n\end{cases}
$$

and

$$
\varphi\left(x_{f}\right)_{p_{i j}}= \begin{cases}t_{n, f(n)} & \text { if }(i, j)=(n, f(n)), \\ 0 & \text { elsewhere. }\end{cases}
$$

$\varphi$ induces a homomorphism $\varphi^{\prime}: A^{\prime}=\left\langle x_{n}, x_{f} \mid n<\omega, f \in \mathscr{F}\right\rangle \rightarrow \widehat{T} / T$. Since $\widehat{T} / T$ is divisible, there is $\varphi^{\prime \prime}: A \rightarrow \widehat{T} / T$ extending $\varphi^{\prime}$. If $a \in A$, $a \notin\left\{x_{n}, x_{f} \mid n<\omega, f \in \mathscr{F}\right\}$, define $\varphi(a)$ to be any element of the coset $\varphi^{\prime \prime}(a)$. Since $A$ is homogeneous of type $0, \varphi$ is an $h$-map, $\varphi: A \rightarrow \widehat{T}$.

Now suppose $\varphi$ lifts to an $h$-map $\psi: B \rightarrow \widehat{T}$. Then $\left|\psi(x)+\varphi\left(x_{n}\right)\right|_{p_{n, 1}}>$ 0 for all $i \geq m_{0}(n)$, i.e., $\psi(x)_{p_{n, 1}} \equiv s_{n i} \bmod p_{n i} \widehat{T}_{p_{n, 1}}$ for $i \geq m_{0}(n)$; on the other hand, define $g: \omega \rightarrow \omega$ via $g(i)=m_{0}(i) \Rightarrow \exists f \in \mathscr{F}$ such that $W=\{i<\omega \mid f(i)=g(i)\}$ is infinite. We have $\left|\psi(x)+\varphi\left(x_{f}\right)\right|_{p_{n, f(n)}} \geq 1$ for $n \geq k_{0}(f) \Rightarrow \psi(x)_{p_{n, f(n)}} \equiv t_{n, f(n)} \bmod p_{n, f(n)} \widehat{T}_{p_{1}}$. Since $\omega$ is infinite, there is $i \in W, i \geq k_{0}(f)$, and for $n \geq k_{0}(f)$ we have

$$
\begin{array}{ll}
\psi(x)_{p_{n, i}} \equiv s_{n i} & \bmod p_{n i} \widehat{T}_{p_{n, i}} \text { and } \\
\psi(x)_{p_{n, i}} \equiv t_{n i} & \bmod p_{n i} \widehat{T}_{p_{n, i}} .
\end{array}
$$

This implies $s_{n i} \equiv t_{n i} \bmod p_{n i} \widehat{T}_{p_{n i}}$, which is a contradiction to the choice of $s_{n i}$ and $t_{n i}$.

\section{SEPARABLE SUBGROUPS OF TORSION-FREE GROUPS}

Our goal in this section is to prove the following

Theorem 5.1. Assume $\mathrm{CH}$ holds. If $G$ is a torsion-free group of cardinality $\aleph_{n}$, $n \leq \omega$, then there is a smooth chain of separable pure subgroups $G_{\alpha}, \alpha<\aleph_{n}$, such that $G=\bigcup_{\alpha<\kappa_{n}} G_{\alpha}$ and $G_{\alpha+1} / G_{\alpha}$ has rank 1 .

We will prove this theorem after some preparation. First we need a

Definition. Let $H$ be a pure subgroup of the torsion-free group $G$. Let $g \in G$. For each countable subset $C$ of $H$, we define a map $g_{c}^{\#}$ from $C$ into the set of all height sequence by $g_{c}^{\#}(x)=|g+x|$ for all $x \in C$. We $H$ hyperbalanced if for each $g \in G$ and for each countable subset $C$ of $H$ there is some $h \in H$ with $g_{c}^{\#}=h_{c}^{\#}$.

Lemma 5.2. Let $0 \neq H$ be a subgroup of $G$. Then there is a hyperbalanced subgroup $L$ of $G$ with $H \subseteq L$ and $|L| \leq|H|^{\aleph_{0}}$.

Proof. By induction on $\alpha<\omega_{1}$ we define a smooth chain of subgroups $H_{\alpha}$ with $H_{0}=H$ and $H_{\lambda}=\bigcup_{\alpha<\lambda} H_{\alpha}$ if $\lambda<\omega_{1}$ is a limit ordinal. Moreover, we will 
have $\left|H_{\alpha}\right| \leq|H|^{\aleph_{0}}$. Suppose $H_{\alpha}$ is already defined. Then $S=\left\{g_{c}^{\#} \mid g \in G, C\right.$ a countable subset of $\left.H_{\alpha}\right\}$ has cardinality $\left|H_{\alpha}\right|^{\aleph_{0}} \cdot\left(2^{\aleph_{0}}\right)^{\aleph_{0}} \leq\left(|H|^{\aleph_{0}}\right) 2^{\aleph_{0}}=|H|^{\aleph_{0}}$. Thus there is a subset $W$ of $G$ with $|W| \leq|H|^{\aleph_{0}}$ and $S=\left\{g_{c}^{\#} \mid g \in W, C\right.$ a countable subset of $H$ \}. Now define $H_{\alpha+1}$ to be the pure subgroup generated by $H_{\alpha}$ and $S$. Note that $\left.\left|H_{\alpha+1 \mid} \leq\right| H\right|^{\aleph_{0}}$. We will show that $L=\bigcup_{\alpha<\omega_{1}} H_{\alpha}$ is hyperbalanced in $G$ : Let $C$ be a countable subset of $L$. Since $c f\left(\omega_{1}\right)=$ $\omega_{1}>\omega$ there is $\alpha<\omega_{1}$ such that $C \subseteq H_{\alpha}$. If $g \in G$ then the construction of $H_{\alpha+1}$ shows the existence of an $h \in H_{\alpha+1} \subseteq L$ with $g_{c}^{\#}=h_{c}^{\#}$. Thus $L$ is hyperbalanced and $|L| \leq \omega_{1} \cdot|H|^{\aleph_{0}}=|H|^{\aleph_{0}}$.

The argument above also shows

Observation 5.3. If $\left\{H_{\alpha}, \alpha<\lambda\right\}$ is a smooth chain of subgroups of $G, c f(\lambda)>$ $\omega$, and each $H_{\alpha+1}$ is hyperbalanced in $G$, then $\bigcup_{\alpha} H_{\alpha}$ is hyperbalanced in $G$.

The following is crucial:

Lemma 5.4. Any hyperbalanced subgroup $H$ of $G$ is balanced in $G$.

Proof. Assumed not. Then there is a coset $0 \neq g+H$ without proper element. By induction on $\alpha<\omega_{1}$ we will construct elements $g_{\alpha} \in g+H$ with $\left|g_{\alpha}\right|<\left|g_{\beta}\right|$ for $\alpha<\beta$. Let $g_{0}$ be any element in $g+H$ and suppose that $g_{\gamma}$ has been defined for all $\gamma<\alpha$.

Note that $C=\left\{g_{\gamma}-g \mid \gamma<\alpha\right\}$ is countable. Since $H$ is hyperbalanced, there is some $h_{1} \in H$ with $g_{c}^{\#}=\left(h_{1}\right)_{c}^{\#}$, i.e., $\left|g+\left(g_{\gamma}-g\right)\right|=\left|h_{1}+\left(g_{\gamma}-g\right)\right|$ for all $\gamma<\alpha$. This implies $\left|g-h_{1}\right| \geq\left|g_{\gamma}\right|$ for all $\gamma<\alpha$. Since there is no proper element in $g+H, g-h_{1}$ is not proper and there is some $h_{2} \in H$ with $\left|g+h_{2}\right| \Varangle\left|g-h_{1}\right|$. Set $C^{\prime}=C \cup\left\{-h_{1}, h_{2}\right\}$ and use again that $H$ is hyperbalanced and we find $h_{3} \in H$ with $\left|g+h_{3}\right| \geq\left|g+h_{2}\right|,\left|g-h_{1}\right|$, and $\left|g_{\gamma}\right|$ for all $\gamma<\alpha$, Now suppose $\left|g+h_{3}\right|=\left|g_{\gamma}\right|$ for some $\gamma<\alpha$. Since $\left|g+h_{3}\right| \geq\left|g-h_{1}\right| \geq\left|g_{\gamma}\right|$ we obtain

$$
\left|g+h_{2}\right| \leq\left|g+h_{3}\right|=\left|g_{\gamma}\right| \leq\left|g-h_{1}\right| \nsucceq\left|g+h_{2}\right|,
$$

a contradiction. This show $\left|g+h_{3}\right|>\left|g_{\gamma}\right|$ for all $\gamma<\alpha$. We may set $g_{\alpha}=g+h_{3}$ and the induction works. This also completes the proof of the lemma, since there is no uncountable ascending chain of height sequences.

Let $G$ be a torsion-free group. By induction on ordinals $\alpha<\omega_{1} \quad\left(=\aleph_{1}\right)$ we define $B^{\alpha}$-subgroups of $G$ : The $B^{0}$-subgroups of $G$ are precisely the balanced subgroups. If $\lambda<\omega_{1}$ is a limit ordinal, then the $B^{\lambda}$-subgroups are all the subgroups of $G$ that are $B^{\alpha}$-subgroups for some $\alpha<\lambda$. The $B^{\alpha+1}$-subgroups are the unions of countable chains of $B^{\alpha}$-subgroups. We call a subgroup $A$ of $G$ a $B^{\infty}$-subgroup if $A$ is a $B^{\alpha}$-subgroup for some $\alpha<\omega_{1}$.

Lemma 5.5. (i) The class of $B^{\infty}$-subgroups is closed with respect to unions of countable chains. 
(ii) If $A$ is a $B^{\infty}$-subgroup of $B$ and $B$ is balanced in $C$, then $A$ is a $B^{\infty}$-subgroup of $C$.

Proof. (i) follows since $c f\left(\omega_{1}\right)>\omega$. To prove (ii) we use induction. If $A$ is a $B^{0}$-subgroup of $B$, i.e., $A$ is balanced in $B$, then $A$ is balanced in $C$ since "balanced" is transitive. We may assume that $A$ is a $B^{\alpha+1}$-subgroup of $B$ and (ii) is true for $B^{\alpha}$-subgroups. Now $A=\bigcup_{n<\omega} A_{n}, A_{n}, B^{\alpha}$-subgroups of $B$. Thus $A_{n}$ are $B^{\infty}$-subgroups of $C$ and $A$ is a $B^{\infty}$-subgroup of $C$.

Theorem 5.6. Assume $C H$ holds. Let $G^{\prime}$ be a torsion-free group such that $G$, $H_{m}, G+H_{m} \quad(m<\omega)$ are balanced subgroups of $G^{\prime}$. Let $|G|=\aleph_{n}, 2 \leq$ $n<\omega$. Then there is an $\aleph_{n}$-filtration $G=\bigcup_{\alpha<\kappa_{n}} G_{\alpha}$ of $G,\left|G_{\alpha}\right|=\aleph_{n-1}$, and $G_{\alpha}\left(G_{\alpha}+H_{m}\right)$ is a $B^{\infty}$-subgroup of $G\left(G+H_{m}\right)$ for all $\alpha$ and $\left|G_{\alpha+1} / G_{\alpha}\right| \leq \aleph_{1}$. If $X$ is a countable subset of $G$, then we may have that $X \subseteq G_{0}$.

Proof. By induction on $n$. We may assume $H_{0}=0$. Suppose $|G|=\aleph_{2}$. Lemma 5.2, Observation 5.3, and a standard back-and-forth argument provide a filtration $G=\bigcup_{\alpha<\aleph_{2}} G_{\alpha}$ where $\left|G_{\alpha}\right|=\aleph_{1}, X \subseteq G_{0}$, and $\left(G_{\alpha}+H_{m}\right) / H_{m}$ is hyperbalanced in $\left(G+H_{m}\right) / H_{m}$ for all $m<\omega$ whenever $c f(\alpha) \neq \omega$. Here we had to use $\mathrm{CH}$ to obtain $\aleph_{n}^{\aleph_{0}}=\aleph_{n}$ in order to avoid possible jumps of our cardinals in 5.2. Since hyperbalanced implies balanced (Lemma 5.4), we have for $c f(\alpha) \neq \omega: G_{\alpha}+H_{m}$ is balanced in $G+H_{m}$. Thus for all $\alpha<\aleph_{2}, G_{\alpha}$ (resp. $\left.G_{\alpha}+H_{m}\right)$ is a $B^{\prime}$-subgroup of $G$ (resp. $\left.G+H_{m}\right)$. Since $\left|G_{\alpha}\right|=\aleph_{1},\left|G_{\alpha+1} / G_{\alpha}\right| \leq$ $\aleph_{1}$ and the theorem holds for $n=2$. Now suppose the theorem holds for $n$. Let $|G|=\aleph_{n+1}$. As before, we get an $\aleph_{n+1}$-filtration $G=\bigcup_{\alpha<\aleph_{n+1}} G_{\alpha}$ where $\left(G_{\alpha}+H_{m}\right) / H_{m}$ are hyperbalanced in $\left(G+H_{m}\right) / H_{m}$ whenever $c f(\alpha) \neq \omega$. Thus $G_{\alpha}+H_{m}$ is balanced in $G+H_{m}$ whenever $c f(\alpha) \neq \omega$. We now show that we can refine this filtration to meet the requirements of our theorem. We distinguish two cases.

(I) $c f(\alpha) \neq \omega$. Here we apply the induction hypothesis where $G_{\alpha+1}$ plays the role of $G$ and $G_{\alpha}, G_{\alpha}+H_{m}$ play the role of $H_{m}$. Thus we find $A_{r}, r<\aleph_{n}$, and $A_{r}$ is a $B^{\infty}$-subgroup of $G_{\alpha+1}+\left(G_{\alpha}+H_{m}\right)$ and $A_{r}+G_{\alpha}$ is a $B^{\infty}$-subgroup of $G_{\alpha+1}+G_{\alpha}$. Now set $G_{\alpha, r}=G_{\alpha}+A_{r}$. Then $G_{\alpha, r}$ is a $B^{\infty}$-subgroup of $G_{\alpha+1}$. and $H_{m}+G_{\alpha, r}$ is a $B^{\infty}$-subgroup of $G_{\alpha+1}+H_{m}$. Since $G_{\alpha+1}, G_{\alpha+1}+H_{m}$ are balanced in $G^{\prime}, H_{m}+G_{\alpha, r}$ (resp. $G_{\alpha, r}$ ) is a $B^{\infty}$-subgroup of $G^{\prime}$ by Lemma 5.5. Since $\left|A_{r+1} / A_{r}\right| \leq \aleph_{1}$ we conclude that also $\left|G_{\alpha, r+1} / G_{\alpha, r}\right| \leq \aleph_{1}$.

(II) $c f(\alpha)=\omega$. Here $G_{\alpha}=\bigcup_{n<\omega} G_{\alpha_{n}}$ and the $\alpha_{n}$ are in case I. We apply the induction hypothesis where $G_{\alpha+1}$ plays the role of $G$ and $G_{\alpha_{n}}, H_{m}+G_{\alpha_{n}}$ play the role of the $H_{m},(m, n) \in \omega \times \omega$. Again, we find an $\aleph_{n}$-filtration $G_{\alpha+1}=\bigcup_{r<\kappa_{n}} A_{r}$ where $\left|A_{r+1} / A_{r}\right| \leq \aleph_{1}, A_{r}+H_{m}+G_{\alpha_{n}}$ is a $B^{\infty}$-subgroup of $G_{\alpha+1}+H_{m}^{n}$, and $A_{r}+G_{\alpha_{n}}$ is a $B^{\infty}$-subgroup of $G_{\alpha+1}$. This implies that $\bigcup_{n<\omega}\left(A_{r}+G_{\alpha_{n}}\right)+H_{m}$ is a $B^{\infty_{n}}$-subgroup of $G_{\alpha+1}+H_{m}$ and $\bigcup_{n<\omega} A_{r}+G_{\alpha_{n}}$ is a $B^{\infty}$-subgroup of $G_{\alpha+1}$. Thus we may set $G_{\alpha, r}=\bigcup_{n<\omega}\left(A_{r}+G_{\alpha_{n}}\right)$ and because of Lemma 5.5, $G_{\alpha, r}$ is a $B^{\infty}$-subgroup of $G^{\prime}$ and $H_{m}+G_{\alpha, r}$ is a $B^{\infty}$-subgroup of 
$G^{\prime}$. Again, since $\left|A_{r+1} / A_{r}\right| \leq \aleph_{1}$, we obtain $\left|G_{\alpha, r+1} / G_{\alpha, r}\right| \leq \aleph_{1}$. This finishes the proof of the theorem.

Corollary 5.7. Assume $C H$ holds. Let $G$ be a torsion-free group, $|G| \leq \aleph_{\omega}$, and $X$ a countable subset of $G$. Then $G$ has a filtration $G=\bigcup_{\alpha<\kappa} G_{\alpha}$ with $G_{\alpha+1} / G_{\alpha}$ torsion-free of rank $1, G_{\alpha}$ separable in $G$, and $X \subseteq G_{0}$, where $G_{0}$ is countable.

Proof. First, let $|G|=\aleph_{n}<\aleph_{\omega}$. If $|G| \leq \aleph_{1}$, there is nothing to show since each countable subgroup is separable. If $|G|=\aleph_{n}$, we have only to use Theorem 5.6 and observe that $B^{\infty}$-subgroups are separable and countable extensions of separable subgroups are again separable. If $|G|=\aleph_{\omega}$, we may use Lemma 5.2, Observation 5.3, and Lemma 5.4 to write $G=\bigcup_{n<\omega} G_{n}, G_{n}$ balanced in $G$, $\left|G_{n}\right|=\aleph_{n}$. We apply Theorem 5.6 to $G_{n+1} / G_{n}$ and find $G_{n} \subset A_{n, r} \subset G_{n+1}$ with $A_{n, r} / G_{n}$ a $B^{\infty}$-subgroup of $G_{n+1} / G_{n}$. We want to show that this implies that $A_{n, r}$ is a $B^{\infty}$-subgroup of $G_{n+1}$.

If $A_{n, r} / G_{n}$ is a $B^{0}$-subgroup of $G_{n+1} / G_{n}$, then $A_{n, r} / G_{n}$ is simply balanced in $G_{n+1} / G_{n}$, and since $G_{n}$ is balanced in $G_{n+1}$, we have that $A_{n, r}$ is balanced. Now assume that $A_{n, r} / G_{n}=\bigcup_{i<\omega}\left(A_{i} / G_{n}\right)$ is a $B^{\alpha+1}$-subgroup of $G_{n+1} / G_{n}$. Our induction implies that $A_{i}$ is a $B^{\infty}$-subgroup of $G_{n+1}$; i.e., $A_{n, r}=\bigcup_{i<\omega} A_{i}$ is a $B^{\infty}$-subgroup of $G_{n+1}$. Thus $G=\bigcup_{n, r} A_{n, r}$ is a filtration into $B^{\infty}$. subgroups of $G$ with $\left|A_{n, r+1} / A_{n, r}\right| \leq \aleph_{1}$. This filtration may be refined to prove the theorem.

Our methods break down if $|G|=\aleph_{\omega+1}$, since one cannot show in general that there are many or even one balanced subgroup of cardinality $\aleph_{\omega}$ of $G$ when $|G|=\aleph_{\omega+1}$, as the following example shows. Again, we assume $\aleph_{n}^{\aleph_{0}}=\aleph_{n}$ for all $n<\omega$, i.e., $\mathrm{CH}$, to obtain $\aleph_{\omega}^{\aleph_{0}}=\aleph_{\omega+1}$. Let $F$ be a free group of rank $\aleph_{\omega}$ and $G$ the cotorsion-hull of $F$. Then $|G|=|F|^{\aleph_{0}}=\aleph_{\omega+1}$. Now suppose $A \subseteq G$ is balanced, $|A|=\aleph_{\omega}$. Since $A$ is pure in $G, \bar{A}$, the cotorsion-hull of $A$, is contained in $G$; i.e., $\bar{A} / A \subset G / A$. Now $\bar{A} / A$ is divisible, which implies $A=\bar{A}$ since $A$ is balanced. Hence $A$ is torsion-free cotorsion of size $\aleph_{\omega}$. In our setting, this is not possible for cardinality reasons.

In conclusion, there are torsion-free groups of cardinality $\aleph_{\omega+1}$ without any balanced subgroups of cardinality $\aleph_{\omega}$ (at least under $\mathrm{CH}$ ). In our example above, $A$ is still separable in $G$ since $\bar{A}$ is a summand of $G$ and $\bar{A} / A$ is divisible. At this time, we do not know of an example of such a $G$ without large separable subgroups. An immediate consequence of Corollary 5.7 and $[\mathrm{AH}, 6.3]$ is the noteworthy

Theorem 5.8. Assume $C H$ holds. If $G$ is a torsion-free group of cardinality $\leq \aleph_{\omega}$, then $\operatorname{Bext}^{2}(G, T)=0$ for any torsion group $T$.

Remark. For $|G|=\aleph_{1}$ this is contained in [AH, Theorem 6.3]. Using the long Hom-Bext sequence established in [Hu], we obtain the following corollary. 
Corollary 5.9. Assume $\mathrm{CH}$ holds. Then any balanced subgroup of a completely decomposable group of cardinality $\leq \aleph_{\omega}$ is a Butler group.

\section{Singular COMPACTNESS}

Here we state a version of Shelah's singular compactness analog to the version in $[\mathrm{H}]$ tailored to our purposes.

Definition 6.1. Let $\lambda$ be a cardinal and $B$ a group. A family $\mathscr{F}$ of subgroups of $B$ is called a $\lambda$-family if

(a) $0 \in \mathscr{F}$.

(b) If $A \in \mathscr{F}$, then $|A|<\lambda$.

(c) If $\mu<\lambda$ and $A_{\alpha}, \alpha<\mu$, is an ascending chain of subgroups in $\mathscr{F}$, then $\bigcup_{\alpha<\mu} A_{\alpha} \in \mathscr{F}$ if for some $\kappa,\left|A_{\alpha}\right| \leq \kappa<\lambda$ for all $\alpha<\mu, \aleph_{0}<\mu$ regular.

(d) If $A \in \mathscr{F}$, and $X \subseteq B$ with $|X|<\lambda$, then there is $C \in \mathscr{F}$ with $|C| \leq|A||X|$ and $A \cup X \subseteq C$.

We adapt a proof in $[\mathrm{H}]$ to prove the next theorem. We give only an outline to indicate where there are changes.

Theorem 6.2 (Hodges/Shelah). Let $\lambda \leq \aleph_{\omega}$ be a singular cardinal, $B$ an abelian group of cardinality $\lambda$, and $\mathscr{F}$ a $\lambda$-family of subgroups of $B$. Let $\mathscr{F}^{*}$ be the family of unions of countable chains of $\mathscr{F}$.

(j) For each $A \in \mathscr{F}^{*}$ there is an axiom 3-family $\mathscr{F}(A)$ of subgroups of $A$; cf. $[\mathrm{AH}]$.

(ij) If $A_{n} \subseteq A_{n+1} \in \mathscr{F}$ for all $n<\omega$ and if $A_{n} \in \mathscr{F}\left(A_{n+1}\right)$ for each $n$, then there are axiom 3-families $\widetilde{F}\left(A_{n}\right)$ of $A_{n}$ and $\widetilde{\mathscr{F}}\left(A_{n}\right) \subseteq \mathscr{F}(A)$ where $A=\bigcup_{n<\omega} A_{n}$, provided $|A|<\lambda$.

(iij) If $\kappa$ is a regular cardinal $<\lambda$ and $C_{i} \in \mathscr{F}, i<\kappa$, is a smooth chain with $\left|C_{i}\right|<\kappa$ for all $i<\kappa$, we set $C=\bigcup_{i<\kappa} C_{i}$ and define $\mathscr{F}(C) \mid C_{i}=\{X \mid$ $\left.X \in \mathscr{F}(C), X \subseteq C_{i}\right\}$. If $C_{i} \in \mathscr{F}(C)$, then $\mathscr{F}(C) \mid C_{i}$ is an axiom 3-subfamily of $\mathscr{F}\left(C_{i}\right)$. Note that $C \in \mathscr{F}$ since $\left|C_{i}\right|<\kappa<\lambda$ and $\kappa$ regular.

Then there is a smooth chain $A^{i}, i<c f(\lambda)$, with $A^{i} \in \mathscr{F}^{*}, A^{i} \in \mathscr{F}\left(A^{i+1}\right)$, and $B=\bigcup_{i<c f(\lambda)} A^{i}$.

Proof. (We adopt the proof in [H] up to some minor changes.) Let $\kappa$ be a regular cardinal $<\lambda$. We define the $\kappa$-Shelah game on $B$ : Player I picks subgroups $B_{i}$ of cardinality $<\kappa$ for $i$ even, $i=0,2,4, \ldots$, and player II picks $B_{i} \in \mathscr{F}$ for $i$ odd such that $B_{i} \subseteq B_{i+1}$ for all $i<\omega$. Player II wins if $B_{i} \in \mathscr{F}\left(B_{i+1}\right)$ for all odd $i$. Observe that $\bigcup_{i<\omega} B_{i} \in \mathscr{F}^{*}$ since $B_{2_{j+1}} \in \mathscr{F}$ and $\left|B_{i}\right|<\kappa<\lambda$. Lemma 1.2 in [H] still holds: the $\kappa$-Shelah game on $B$ is determinate; i.e., if player I has no winning strategy, then II has one! Thus, we have only to show that player I has no winning strategy. By way of contradiction, let us assume I has a winning strategy $s$. We have to show that II can defeat $s$. Let's play! Player I moves first and picks $B_{0}$. Now player II (he's got all the time in the world) gets working. By transfinite induction he constructs a 
smooth chain $C_{i}(i<\kappa)$ of subgroups of $B$ with $C_{i} \in \mathscr{F}^{*}, C_{i} \in \mathscr{F}$ if $(i) \neq \omega$, and $\left|C_{i}\right|<\kappa$ for all $i<\kappa$ such that: For each $j>\kappa$ and each finite set of ordinals $j_{1}<\cdots<j_{n}<j$ each subgroup showing up in an initial piece (of length $2 n$ ) of the $\kappa$-Shelah game where I plays his strategy $s$ and starts with $B_{0}$ and II plays $C_{j_{1}}, \ldots, C_{j_{n}}$ is contained in $C_{i}$. This can be done since $j<\kappa, \kappa$ regular and (d). Because (c), $C=\bigcup_{i<\kappa} C_{i} \in \mathscr{F}$. Since there is the axiom 3-family $\mathscr{F}(C)$ on $C$, we may assume-passing to $a$ on $b \subseteq \kappa$-that $C_{i} \in \mathscr{F}(C)$ for all $i<\kappa$. Thus $C_{i} \in \mathscr{F}(C)\left|C_{i} \subseteq \mathscr{F}(C)\right| C_{i+1} \subseteq \mathscr{F} C\left(C_{i+1}\right)$, i.e., $C_{i} \in \mathscr{F}\left(C_{i+1}\right)$.

Now II easily defeats I by choosing $B_{i+1}$ to be $C_{\alpha(i)}$ where $\alpha(i)=1+\inf \{\alpha<$ $\left.\kappa \mid B_{0}, B_{1}, \ldots, B_{i} \subseteq C_{\alpha}\right\}$. Now let $\left(\kappa_{i}\right), i<c f(\lambda)$, be a strictly increasing sequence of regular cardinals, $\sup \left\{\kappa_{i} \mid i<c f(\lambda)\right\}=\lambda$ and $\kappa_{0}>c f(\lambda)$. Since $\mathscr{F}$ is a $\lambda$-family on $B$, we may write $B=\bigcup_{i<c f(\lambda)} C^{i},\left|C^{i}\right|=\kappa_{i}, C^{i} \in$ $\mathscr{F}$. We want to extend $C^{i}$ to $A^{i}, i<c f(\lambda)$, smooth chain, $A^{i} \in \mathscr{F}^{*}$, and $A^{i} \in \mathscr{F}\left(A^{i+1}\right)$ for all $i<c f(\lambda)$. Each $A^{i}$ will be the union of a chain $C^{i}=C_{0}^{i} \subseteq B_{0}^{i} \subseteq A_{0}^{i} \subseteq C_{1}^{i} \subseteq B_{1}^{i} \subseteq A_{1}^{i}, \subseteq \cdots$ with

(i) If $i-1$ exists, then $C_{0}^{i}, B_{0}^{i}, C_{1}^{i} \subseteq B_{1}^{i}, \ldots$ is a play of the $\kappa_{i}^{+}$-Shelah game on $B$ where II uses his winning strategy picking the $B_{k}^{i}$ 's (I picks the $C_{k}^{i}$ 's). If $i$ is a limit or 0 , set $B_{k}^{i}=C_{k}^{i}$ for all $k<\omega$. We make player I (each upper index stands for a separable game) pick the $C_{k}^{i}$ 's. (As in [H], the $A_{n}^{i}$ will be defined and used later to ensure that the chain $\left\{A^{i}\right\}$ is smooth.) We also make I pick the $C_{k}^{i}$ 's such that:

(ii) Whenever $A_{n}^{j}$ is defined for each $j<c f(\lambda)$, then $C_{n+1}^{i} \supseteq \bigcup_{j \leq i} A_{n}^{j}$ and for $k=0,1,2, \ldots, n C_{n+1}^{i} \cap B_{k}^{i+1} \in \widetilde{F}\left(B_{k}^{i+1}\right)$ where $\widetilde{\mathscr{F}}\left(B_{k}^{i+1}\right) \subseteq \mathscr{F}\left(\bigcup_{k<\omega} B_{k}^{i}\right)$ is the family in hypothesis (ij). This can be done by using a standard back-andforth argument using the fact that $\widetilde{F}\left(B_{k}^{i+1}\right)$ is closed with respect to (countable) unions.

Now define $A^{i}=\bigcup_{n<\omega} A_{n}^{i}=\bigcup_{n<\omega} C_{n}^{i}=\bigcup_{n<\omega} B_{n}^{i}$. We have $A^{i}=A^{i} \cap$ $A^{i+1}=A^{i} \cap\left(\bigcup_{k<\omega} B_{k}^{i+1}\right)=\bigcup_{k<\omega}\left(A^{i} \cap B_{k}^{i+1}\right)=\bigcup_{k<\omega}\left(\left(\bigcup_{k<n<\omega} C_{n}^{i}\right) \cap B_{k}^{i+1}\right)=$ $\bigcup_{k<\omega}\left(\bigcap_{k<n<\omega}\left(C_{n}^{i} \cap B_{k}^{i+1}\right)\right)$. Since $C_{n}^{i} \cap B_{k}^{i+1} \in \widetilde{\mathscr{F}}\left(B_{k}^{i+1}\right)$ for all $k \leq n$, we conclude $C_{n}^{i} \cap B_{k}^{i+1} \in \mathscr{F}\left(A^{i+1}\right), A^{i+1}=\bigcup_{k<\omega} B_{k}^{i+1}$, and since $\mathscr{F}\left(A^{i+1}\right)$ is closed with respect to countable unions of subgroups of size $\kappa_{i}$, we conclude that $A^{i} \in \mathscr{F}\left(A^{i+1}\right)$. Now we may complete the proof as in [H, p. 210] by defining the $A_{n}^{i}$ 's to ensure that $\left\{A^{i} \mid i<c f \lambda\right\}$ is smooth.

We are interested in

Corollary 6.3. Let $B$ be a Butler group of singular cardinality $\lambda$ and assume that $B$ admits to a $\lambda$-family $\mathscr{F}$ of $B_{2}$-subgroups. Then $B$ is a $B_{2}$-group.

Proof. For $A \in \mathscr{F}$ we let $\mathscr{F}(A)$ be an axiom 3-family of decent subgroups as introduced in $[\mathrm{AH}]$. We have to show that 6.2 applies: (j) takes care of itself. To verify (ij), let $A_{n} \subset A_{n+1}, n<\omega$, be a chain in $\mathscr{F}$ and $A_{n} \in \mathscr{F}\left(A_{n+1}\right)$. Thus 
$A_{n}$ is decent in $\left(A_{n+1}\right)$ and-better-shows up in a $B_{2}$-filtration of $A_{n+1}$. Thus $A=\bigcup_{\alpha<\mu} H_{\alpha}=\bigcup_{n<\omega} A_{n}, H_{\alpha+1}=H_{\alpha}+G_{\alpha}, G_{\alpha}$ pure of finite rank and there is a sequence $\alpha_{n}<\alpha_{n+1}<\mu$ with $\mu=\sup \left\{\alpha_{n} \mid n<\omega\right\}$ and $H_{\alpha_{n}}=A_{n}$. Use this $B_{2}$-filtration of $A$ and the $A_{n}$ 's to define "closed" subsets of $\mu$ and $\alpha_{n}$ as in [AH]. This gives rise to an axiom 3-family $\widetilde{F}(A) \subseteq \mathscr{F}(A)$, $\widetilde{\mathscr{F}}(A) \mid A_{n}=\mathscr{F}\left(A_{n+1}\right) \subseteq \mathscr{F}\left(A_{n+1}\right)$, and since $S \subseteq \alpha_{n}$ is "closed" iff $S$ is closed in $\mu, \widetilde{\mathscr{F}}\left(A_{n}\right) \subseteq \widetilde{\mathscr{F}}(A) \subseteq \mathscr{F}(A)$. This shows that (ij) holds. Let the $C_{i}$ 's in (iij) play the role of the $A_{n}$ and (iij) follows.

\section{THE STRUCTURE OF BUtLER GROUPS}

We will give a cardinal induction to show that under $\mathrm{CH}$ every Butler group of cardinality $\leq \aleph_{\omega}$ is a $B_{2}$-group. First we want to cover the "regular case."

Theorem 7.1. Let $G$ be a Bulter group of regular cardinality $\kappa$ such that $G$ has a filtration $G=\bigcup_{\alpha<\kappa} G_{\alpha}$ and each $G_{\alpha}$ is a Butler group with $\left|G_{\alpha}\right|<\kappa$. Then there is a cub $C \subseteq \kappa$ and for any $\alpha \in C$, we have that $G_{\alpha}$ has the TEP in each $G_{\beta}, \beta \geq \alpha$.

Proof. Let $E=\left\{\alpha \in \kappa \mid\right.$ There is a $\alpha<\beta<\kappa$ such that $G_{\alpha}$ does not have TEP in $\left.G_{\beta}\right\}$. By way of contradiction we assume that $E$ is stationary in $\kappa$. We may assume that if $\alpha \in E$, then $G_{\alpha}$ does not have the TEP in $G_{\alpha+1}$. Thus there exist a ( $\Sigma$-cyclic) torsion group $T_{\alpha}$ of cardinality $\leq\left|G_{\alpha+1}\right|$ and a homomorphism $\varphi_{\alpha}: G_{\alpha} \rightarrow T_{\alpha}$ that does not lift to $G_{\alpha+1}$. Now define $S_{\alpha}=\bigoplus_{\beta<\alpha} T_{\beta}$. (Here we borrow an idea from $[\mathrm{EF}]$.) By transfinite induction we construct a direct system $\left\{M_{\alpha}(\alpha<\kappa) ; \Pi_{\alpha}^{\beta}\right\} \quad([\mathrm{F} 1$, p. $53 \mathrm{ff}])$ as follows: For any $\alpha<\beta<\kappa$ we have

(1) $M_{\alpha}$ is a mixed group with torsion subgroup $S_{\alpha}$ and $M_{\alpha} / S_{\alpha} \cong G_{\alpha}$.

(2) $0 \rightarrow S_{\alpha} \rightarrow M_{\alpha} \rightarrow G_{\alpha} \rightarrow 0$ is balanced exact.

(3) All $\Pi_{\alpha}^{\beta}$ are embeddings and $\Pi_{\alpha}^{\beta} \mid S_{\alpha}=$ id.

(4) If $\lambda$ is a limit ordinal, then $M_{\lambda}={\underset{\lim }{\longrightarrow}}_{\lambda} M_{\alpha}$ and $\Pi_{\alpha}^{\lambda}: M_{\alpha} \rightarrow M_{\lambda}, \alpha<\lambda$, are the natural maps induced by $\Pi_{\alpha}^{\beta}, \alpha<\beta<\lambda$.

(5) For all $\alpha<\beta$ we have $\Pi_{\alpha}^{\beta}\left(M_{\alpha}\right)+S_{\beta}=\Pi_{\alpha}^{\beta}\left(M_{\alpha}\right) \oplus S_{\beta, \alpha}$ where $S_{\beta, \alpha}=$ $\bigoplus_{\alpha \leq r<\beta} T_{r}$.

Note that (5) implies that for any limit ordinal $\lambda$ and $\alpha<\lambda, \Pi_{\alpha}^{\lambda}\left(M_{\alpha}\right)+S_{\lambda}=$ $\Pi_{\alpha}^{\lambda}\left(M_{\alpha}\right) \oplus S_{\lambda, \alpha}$ and therefore $S_{\lambda}$ is balanced in $M_{\lambda}$ since $\Pi_{\alpha}^{\lambda}\left(S_{\alpha}\right)$ is balanced in $\Pi_{\alpha}^{\lambda}\left(M_{\alpha}\right)$. (Keep in mind that all $\Pi_{\alpha \alpha}^{\lambda}$ are embedding.)

(6) If $M_{\alpha}$ is already defined and $\alpha \in E$, then $M_{\alpha}=S_{\alpha} \oplus B_{\alpha}$ and $B_{\alpha} \cong$ $G_{\alpha}$. Let $\mu_{\alpha}: G_{\alpha} \rightarrow B_{\alpha}$ be an isomorphism. This isomorphism extends to an isomorphism $\mu_{\alpha+1}: G_{\alpha+1} \rightarrow B_{\alpha+1}$ where $B_{\alpha+1} \supseteq B_{\alpha}$. We set $M_{\alpha+1}=$ $S_{\alpha+1} \oplus B_{\alpha+1}$ and define $\Pi_{\alpha}^{\alpha+1}(s, b)=\left(s+\varphi_{\alpha}\left(\mu_{\alpha}^{-1}(b)\right), b\right)$ for all $s \in S_{\alpha}, b \in B_{\alpha}$. Clearly, $\Pi_{\alpha}^{\alpha+1}: M_{\alpha} \rightarrow M_{\alpha+1}$ is an embedding.

If $\alpha \notin E$, we define $\Pi_{\alpha}^{\alpha+1}$ as above with $\varphi_{\alpha}=0$. We have to show that (5) 
is satisfied. Let $\beta \leq \alpha$. Then

$$
\begin{aligned}
S_{\alpha+1}+\Pi_{\beta}^{\alpha+1}\left(M_{\beta}\right) & =\Pi_{\alpha}^{\alpha+1}\left(\Pi_{\beta}^{\alpha}\left(M_{\beta}\right)\right)+S_{\alpha+1} \subseteq M_{\alpha}+S_{\alpha+1} \\
& =M_{\alpha}+S_{\alpha+1, \alpha}=\left(M_{\alpha}+S_{\alpha}\right) \oplus S_{\alpha+1, \alpha} .
\end{aligned}
$$

By induction hypothesis, $\Pi_{\beta}^{\alpha}\left(M_{\beta}\right)+S_{\alpha}=\Pi_{\beta}^{\alpha}\left(M_{\beta}\right)+S_{\alpha, \beta}$. Thus $S_{\alpha+1}+\Pi_{\beta}^{\alpha+1}\left(M_{\beta}\right)$ $=\Pi_{\beta}^{\alpha}\left(M_{\beta}\right) \oplus S_{\alpha, \beta} \oplus S_{\alpha+1, \alpha}=\Pi_{\beta}^{\alpha}\left(M_{\beta}\right) \oplus S_{\alpha+1, \beta}$. If $\lambda$ is a limit and $\beta<\lambda$, then

$$
\begin{aligned}
\Pi_{\beta}^{\lambda}\left(M_{\beta}\right)+S_{\lambda} & =\Pi_{\beta}^{\lambda}\left(M_{\beta}\right)+S_{\lambda, \beta}=\bigcup_{\beta \leq \alpha<\lambda}\left(\Pi_{\beta}^{\lambda}\left(M_{\beta}\right) \oplus S_{\alpha, \beta}\right) \\
& =\Pi_{\beta}^{\lambda}\left(M_{\beta}\right) \oplus \bigcup_{\beta \leq \alpha<\lambda} S_{\alpha, \beta}=\Pi_{\beta}^{\lambda}\left(M_{\beta}\right) \oplus S_{\lambda, \beta} .
\end{aligned}
$$

Let $M_{k}=\underset{\lim _{\kappa}}{\longrightarrow} M_{\alpha}$. Then $M_{\kappa}=\bigcup_{\alpha<\kappa} \Pi_{\alpha}^{k}\left(M_{\alpha}\right)$ and $\Pi_{\alpha}^{\kappa}\left(M_{\alpha}\right)+S_{\kappa}=\Pi_{\alpha}^{\kappa}\left(M_{\alpha}\right) \oplus$ $S_{\kappa, \alpha}$. Therefore $S_{\kappa}=\bigoplus_{\alpha<\kappa} T_{\alpha}$ is balanced in $M_{\kappa}$ with $M_{\kappa} / S_{\kappa} \cong G$. Since $G$ is a Butler group, there is a splitting map $\psi: G \rightarrow M_{\kappa}$. Since $\kappa$ is regular and $\left|M_{\alpha}\right|<\kappa$ for all $\alpha$, there is a cub $C \subseteq \kappa$ such that $\psi\left(G_{\alpha}\right) \subseteq \Pi_{\alpha}^{\lambda}\left(M_{\alpha}\right)$ for all $\alpha \in C$. Since $E$ is stationary, there is some $\alpha \in E \cap C$. Let $\beta \in C$ with $\beta>\alpha$. Then $\psi \mid G_{\beta}: G_{\beta} \rightarrow \Pi_{\beta}^{\lambda}\left(M_{\beta}\right)$ is a splitting map. We may apply $\left(\Pi_{\beta}^{\lambda}\right)^{-1}$ and we may assume that $\psi: G_{\beta} \rightarrow M_{\beta}$ and $\psi \mid G_{\alpha} \rightarrow \Pi_{\alpha}^{\beta}\left(M_{\alpha}\right)$ is a splitting map. We have $\psi(g)=\left(h(g), \mu_{\beta}(g)\right)$ for all $g \in G_{\beta}$ and $h=h_{0} \oplus h_{1}: G_{\beta} \rightarrow$ $S_{\alpha+1} \oplus S_{\beta, \alpha+1}, h_{1} \mid G_{\alpha}=0$. If $g \in G_{\alpha}$, then $h(g) \in S_{\alpha}$. We may subtract $h_{1}$ from $\psi$ and thus are able to assure $h_{1}=0$. Then $\psi: G_{\alpha+1} \rightarrow M_{\alpha+1}$ is a splitting map and

$$
\begin{aligned}
\Pi_{\alpha}^{\alpha+1}\left(M_{\alpha}\right)= & \left.\left\{s+\varphi_{\alpha}\left(\mu_{\alpha}^{-1}(b)\right), b\right) \mid b \in B_{\alpha}, s \in S_{\alpha}\right\} \subseteq S_{\alpha} \\
& \oplus T_{\alpha} \oplus\left\{\left(h\left(\mu_{\alpha}^{-1}(b)\right), b\right) \mid b \in B_{\alpha}\right\}
\end{aligned}
$$

and $\Pi_{\alpha}^{\alpha+1}\left(M_{\alpha}\right)=S_{\alpha} \oplus\left\{h\left(\mu_{\alpha}^{-1}(b), b\right) \mid b \in B_{\alpha+1}\right\}$. Thus $\left.\varphi_{\alpha}\left(\mu_{\alpha}^{-1}(b)\right), b\right) \equiv$ $h\left(\mu_{\alpha}^{-1}(b)\right) \bmod S_{\alpha}$ for all $b \in B_{\alpha}$. Let $\Pi: S_{\alpha+1} \rightarrow T_{\alpha}$ be the projection. Then $\Pi \circ \varphi_{\alpha}=\varphi_{\alpha}$ and $\varphi_{\alpha}\left(\mu_{\alpha}^{-1}(b)\right)=(\Pi \circ h)\left(\mu_{\alpha}^{-1}(b)\right)$ for all $b \in B_{\alpha}$. This implies $\varphi_{\alpha}=(\Pi \circ h) \mid G_{\alpha}$ and $\varphi_{\alpha}$ lifts to $G_{\alpha+1}$, a contradiction. This shows that $E$ is not stationary and therefore we may assume that $G=\bigcup_{\alpha<\kappa} G_{\alpha}$ is a filtration where each $G_{\alpha}$ has TEP in $G_{\alpha+1}$.

Next we will modify Lemma 3.1 in [AH]. We refer to [AH] for a definition of Hill's compatibility relation $\|$.

Lemma 7.2. Let $A, B$, and $H$ be pure subgroups of the torsion-free group $G$.

(a) If $A$ is a balanced in $G$ and $A \| H$, then $A \cap H$, is balanced in $H$.

(b) If $A \| H$ and $H+A \| B$ then $H \| A+B$.

Proof. To prove (a), let $h \in H-(H \cap A)=H-A$. For any $a \in H \cap A \subseteq A$ we have $|h+a| \leq\left|h+a_{0}\right|$ where $a_{0}=a_{0}(h) \in A$ since $A$ is balanced in $G$. There is some element $a_{1} \in A \cap H$ such that $|h+a| \leq\left|h+a_{0}\right| \leq\left|h+a_{1}\right|$ for all $a \in A \cap H$. Thus $A \cap H$ is balanced in $H$. To show (b), fix some 
$h \in H, a \in A, b \in B$. Then $|h+a+b|=|(h+a)+b| \leq\left|h+a+b_{0}\right|$ where $b_{0} \in(H+A) \cap B$, i.e., $b_{0}=h_{0}+a_{0} \in B$ and $h_{0} \in H, a_{0} \in A$. Thus $|h+a+b| \leq\left|h+a+h_{0}+a_{0}\right| \leq\left|\left(h+h_{0}\right)+\left(a+a_{0}\right)\right| \leq\left|h+h_{0}+x\right|$ for some $x \in A \cap H$ since $H \| A$. This implies $|h+a+b| \leq\left|h+\left(b_{0}-a_{0}\right)+x\right|$ and since $a_{0}, x \in A, b_{0} \in B$ we have $y=b_{0}-a_{0}+x=h_{0}+x \in(A+B) \cap H$. This shows $H \| A+B$.

Definition $7.3[\mathrm{FH}]$. Let $G$ be a group, $\lambda$ a cardinal, and $\mathscr{F}$ a family of subgroups of $G$. We call $\mathscr{F}$ a $G(\lambda)$-family if

(a) $0 \in \mathscr{F}$.

(b) If $\left\{F_{i} \mid i \in I\right\} \subseteq \mathscr{F}$ is a chain then $\bigcup_{i \in I} F_{i} \in \mathscr{F}$.

(c) If $F \in \mathscr{F}$ and $A \subseteq G,|A| \leq \lambda$, then there is $F^{\prime} \in \mathscr{F}$ with $A+F / F \subseteq$ $F^{\prime} / F$ and $\left|F^{\prime} / F\right| \leq \lambda$.

Definition 7.4. Let $C=\bigoplus_{i \in I} R_{i}$ be a completely decomposable group. For any subset $J \subseteq I$ we set $C(J)=\bigoplus_{i \in J} R_{i}$. If $H$ is a subgroup of $C$, we call a subset $J \subseteq I$ an $H$-special set if $C(J) \| H$. Moreover, we define $H(J)=$ $H \cap C(J)$.

Theorem 7.5. Let $H$ be a pure subgroup of the completely decomposable group $C=\bigoplus_{i \in I} R_{i}$. Then $H$ admits a $G\left(2^{\aleph_{0}}\right)$-family $\mathscr{F}_{H}$ of balanced subgroups induced by a $G\left(2^{\aleph_{0}}\right)$-family $\mathscr{F}_{c}$ of direct summands of $C$, i.e., $\mathscr{F}_{H}=\{X \cap H \mid$ $\left.X \in \mathscr{F}_{c}\right\}$.

Proof. Let $\mathscr{F}_{H}$ be the family of $H$-special subsets of $I$ and $\mathscr{F}_{H}^{\#}=\{C(X) \mid X \in$ $\mathscr{F}_{H}$ \}. We want to show that $\mathscr{F}_{H}^{\#}$ is a $G\left(2^{\aleph_{0}}\right)$ family of $C$. We have only to show 7.3(c). So let $C(J) \in \mathscr{F}_{h}^{\#}$ and $A \subseteq C,|A| \leq 2^{\aleph_{0}}$. By induction on $n<\omega$ we define subsets $Z_{n} \subseteq I,\left|Z_{n}\right| \leq 2^{\aleph_{0}}$ : Let $Z_{0}$ be minimal with $A \subseteq C\left(Z_{0}\right)$. Suppose $Z_{n}$ is already defined. For $g \in C\left(Z_{n}\right)$ we find a set $\left\{h_{\alpha, g} \mid \alpha<2^{\aleph_{0}}\right) \subseteq$ $H+C(J)$ such that for any $y \in H+C(J)$ we have $|y+g| \leq\left|h_{\alpha, g}+g\right|$ for some $\alpha<2^{\aleph_{0}}$. (Note that there are only $2^{\aleph_{0}}$ many height sequences.) Now let $Z_{n+1}$ be minimal with $C\left(Z_{n}\right) \cup\left\{h_{\alpha, g} \mid g \in C\left(Z_{n}\right), \alpha<2^{\aleph_{0}}\right\} \subseteq C\left(Z_{n+1}\right)$. Note that $\left|Z_{n+1}\right| \leq 2^{\aleph_{0}}$. Let $Z=\bigcup_{n<\omega} Z_{n}$. Then $H+C(J) \| C(Z)$ and $H \| C(J)$ since $J \in \mathscr{F}_{H}$. By 7.2(b), $H \| C(J)+C(Z)=C(J \cup Z)$ and by 7.2(a), $H\left(J^{\#}\right)=H \cap C\left(J^{\#}\right), J^{\#}=J \cup Z$ is a balanced subgroup of $H$. Now we are done since $\left|J^{\#}-J\right| \leq|Z| \leq 2^{\aleph_{0}}$.

Corollary 7.6 (CH). Let $H$ be a balanced subgroup of a completely decomposable group such that $H$ is a Butler group. Then $H$ admits a $G\left(\aleph_{1}\right)$-family of Butler groups.

Proof. Observe that a countable extension of a balanced subgroup of $H$ is separable in $H$ and Corollary 4.6.

We are now ready to prove our main theorem. 
Main Theorem (7.7). Assume $\mathrm{CH}$ holds. Then any Butler group of cardinality $\leq \aleph_{\omega}$ is a $B_{2}$-group.

Proof. By cardinal induction. Let $G$ be a Butler group of cardinality $\kappa$ and assume that each Butler group of cardinality $<\kappa \leq \aleph_{\omega}$ is a $B_{2}$-group. Note that $\kappa=\aleph_{1}$ has this property. Let $0 \rightarrow H \rightarrow C \rightarrow G \rightarrow 0$ be a balanced resolution of $G$; i.e., $C$ is completely decomposable and $H$ is balanced in $C$. We may assume that $|H|=|C|=\kappa$. By Theorem 7.5 there is a $G\left(\aleph_{1}\right)$-family $\mathscr{F}_{c}$ of summands of $C$ such that $\mathscr{F}_{H}=\left\{H \cap X \mid X \in \mathscr{F}_{c}\right\}$ is a $G\left(\aleph_{1}\right)$-family of balanced subgroups of $H$. Therefore we may-for any $S \in \mathscr{F}_{H}$-write $H=\bigcup_{\alpha<\kappa} H_{\alpha}$ with $H_{\alpha} \in \mathscr{F}_{H}$ and $\left|H_{\alpha+1} / H_{\alpha}\right| \leq \aleph_{1}$ and $H_{0}=S$. Since $|G| \leq \aleph_{\omega}$, Theorem 5.8 implies that $\operatorname{Bext}^{2}(G, T)=0$. Since $H$ is balanced in $C$, the long Hom-Bext sequence implies that $\operatorname{Bext}(H, T)=0$; i.e., $H$ is a Butler group. By Corollary 4.6 we obtain that each $H_{\alpha}$ is also a Butler group; i.e., any $S \in \mathscr{F}_{H}$ is a Butler group and by induction hypothesis, $H$ has a $G\left(\aleph_{1}\right)$-family $\mathscr{F}_{H}$ of $B_{2}$-groups.

If $\kappa \leq \aleph_{\omega}$ is singular, Corollary 6.3 implies that $H$ is a $B_{2}$-group. If $\kappa$ is regular we may apply Theorem 7.1 and there is a filtration $H=\bigcup_{\alpha<\kappa} H_{\alpha}$ with $H_{\alpha} \in \mathscr{F}_{H},\left|H_{\alpha}\right|<\kappa$, and each $H_{\alpha}$ has TEP in $H_{\alpha+1}$ Since $H_{\alpha+1}$ is a pure subgroup of $C$, each pure finite-rank subgroup of $H_{\alpha+1}$ is a Butler group of finite rank. Moreover, $H_{\alpha+1} / H_{\alpha}$ is a Butler group of cardinality $<\kappa$ by Corollary 4.3. Thus we may apply Proposition 3.9 and continue any $B$-filtration of $H_{\alpha}$ to one of $H_{\alpha+1}$. This and an obvious induction over $\alpha<\kappa$ show that $H$ is a $B_{2}$-group. By Corollary 3.5 we may assume that each $A \in \mathscr{F}_{H}$ has the TEP in $H$. Recall $A=H \cap X, X \in \mathscr{F}_{c}$. If $X \in \mathscr{F}_{c}$ such that $X \cap H$ has TEP in $H$, then $X \cap H$ has TEP in $C$ since $H$ does. Therefore $X / X \cap H \cong X+H / H$ is a Butler group and we may assume that $G$ admits a $G\left(\aleph_{1}\right)$-family $\mathscr{F}_{G}$ of Butler groups, namely $\mathscr{F}_{G}=\left\{X+H / H\left|X \in \mathscr{F}_{c},\right| X \mid<\kappa\right\}$. By induction hypothesis, all groups in $\mathscr{F}_{G}$ are $B_{2}$-groups. If $\kappa$ is singular, $G$ is a $B_{2}$-group by Corollary 6.3. Thus we may assume that $\kappa$ is regular. Again, Theorem 7.1 and properties of $\mathscr{F}_{G}$ show the existence of a filtration $G=\bigcup_{\alpha<K} G_{\alpha}$ into $B_{2}$ groups $G_{\alpha},\left|G_{\alpha}\right|<\kappa$ and each $G_{\alpha}$ has the TEP in $G_{\alpha+1}$. By Theorem 3.7(i), each pure finite-rank subgroup of $G$ is Butler since $H$ is a $B_{2}$-group. In the same way as above for $H$, we utilize 4.3 and 3.7 to show that $G$ is a $B_{2}$-group.

\section{REFERENCES}

[A] D. Arnold, Notes on Butler groups and balanced extensions, Boll. Un. Math. Ital. A (6) 5 (1986), 175-184.

[AH] U. Albrecht and P. Hill, Butler groups of infinite rank and axiom 3, Czechoslovak Math. J. 37 (112) (1987), 293-309.

[B] L. Bican, Splitting in mixed groups, Czechoslovak Math. J. 28 (1978), 356-364.

[BS] L. Bican and L. Salce, Infinite rank Butler groups, Proc. of Abelian Group Theory Conference, Honolulu, Lecture Notes in Math., vol. 1006, Springer-Verlag, Berlin, 1983, pp. 171-189.

[BSS] L. Bican, L. Salce and J. Stepan, A characterization of countable Butler groups, Rend. Sem. Mat. Univ. Padova 74 (1985), 51-58. 
[DR1] _ On torsion-free abelian k-groups, Proc. Amer. Math. Soc. 99 (1987), 403-408.

[DR2] _ Infinite rank Butler groups, Trans. Amer. Math. Soc. (to appear).

[D] M. Dugas, On some subgroups of infinite rank Butler groups, Rend. Sem. Mat. Univ. Padova 79 (1988), 153-161.

[E] P. Eklof, Set theoretic methods in homological algebra and abelian group, Presses Univ. Montreal, Montreal, 1980.

[EF] P. Eklof and L. Fuchs, Baer modules over valuation domains, Ann. Mat. Pura Appl. 150 (1988), 363-373.

[Fu] L. Fuchs, Infinite abelian groups, vols. I and II, Academic Press, New York, 1977 and 1973.

[FH] L. Fuchs and P. Hill, The balanced-projective dimension of abelian p-groups, Trans. Amer. Math. Soc. 293 (1986), 99-112.

[HM] P. Hill and C. Megibben, Torsion-free groups, Trans. Amer. Math. Soc. 295 (1986), 735-751.

[H] W. Hodges, In singular cardinality, locally free algebras are free, Algebra Universalis 12 (1981), 205-220.

[Hu] R. Hunter, Balanced subgroups of abelian groups, Trans. Amer. Math. Soc. 215 (1976), 81-98.

[S] S. Shelah, A compactness theorem for singular cardinals, free algebras, Whitehead problem and transversals, Israel J. Math. 21 (1975), 319-349.

Department of Mathematics, Baylor University, Waco, Texas 76793

Department of Mathematics, Auburn University, Auburn, Alabama 36849

Department of Mathematics, University of Colorado, Colorado Springs, Colorado 80933-7150 